\title{
Fusión de identidad: Una revisión sistemática
}

\author{
Diego Henríquez; Alfonso Urzúa; Wilson López-López
}

\begin{abstract}
How to cite this article:
Henríquez, D., Urzúa, A., \& López-López, W. (2020). Identity fusion: A systematic review. Acta Colombiana de Psicología, 23(2), 383-409. http://www.doi.org/10.14718/ACP.2020.23.2.15
\end{abstract}

Recibido, abril 26/2019; Concepto de evaluación, marzo 10/2020; Aceptado, abril 13/2020

\author{
Diego Henríquez \\ Universidad Católica del Norte, Antofagasta, Chile \\ ORCID: https://orcid.org/0000-0003-4282-4246 \\ Alfonso Urzúa* \\ Universidad Católica del Norte, Antofagasta, Chile \\ ORCID: https://orcid.org/0000-0002-0882-2194 \\ Wilson López-López \\ Pontificia Universidad Javeriana, Bogotá, Colombia \\ ORCID: https://orcid.org/0000-0002-2964-0402
}

\begin{abstract}
Resumen
El objetivo de la presente investigación fue realizar una revisión sistemática de los estudios empíricos que han reportado el uso de la teoría de fusión de identidad. Para esto, la revisión siguió los lineamientos y recomendaciones de la declaración PRISMA. Las bases de datos consultadas fueron Web of Science, Scopus, ProQuest, ScienceDirect, Willey Online Library, EBSCO y JSTORE. En total, se revisaron 52 estudios empíricos, en español y en inglés, publicados entre los años 2009 y 2018, que cumplían con los criterios de selección. La mayoría de los estudios dan evidencia de que la fusión de identidad es un fuerte predictor de conductas extremas de sacrificio por el grupo, y también, se ha asociado generalmente a variables como la identificación con el grupo, el compromiso grupal, el apoyo social percibido, la lealtad incluso en condiciones de ostracismo, los sentimientos, afectos y emociones hacia el grupo, y la percepción de parentesco, entre otras. Al final se discuten sus implicaciones y limitaciones.

Palabras clave: fusión de identidad, sacrificio extremo, identidad grupal, apoyo social.
\end{abstract}

\section{Identity fusion: A systematic review}

\begin{abstract}
The objective of the present investigation was to conduct a systematic review of empirical studies that have reported the use of identity fusion theory. The review followed guidelines and recommendations of the PRISMA statement. The following databases were used Web of Science, Scopus, ProQuest, ScienceDirect, Willey Online Library, EBSCO and JSTORE databases. Empirical studies were selected, in Spanish and English that were published between 2009 and 2018. 52 articles were found that met the selection criteria. Most studies give evidence that identity fusion is a strong predictor of extreme sacrifice behaviors by the group. Likewise, identity fusion has generally been associated with variables such as: identification with the group, group commitment, perceived social support, loyalty even in ostracized conditions, feelings, affects and emotions towards the group, perception of kinship, among other. Its implications and limitations are discussed.

Keywords: identity fusion, extreme sacrifice, group identity, social support.
\end{abstract}

* Escuela de Psicología, Universidad Católica del Norte, Angamos, n. ${ }^{\circ} 0610$, Antofagasta, Chile. alurzua@ucn.cl Esta publicación se deriva del proyecto FONDECYT Regular \#1180315, financiado por la Comisión Nacional de Investigación Científica y Tecnológica del Gobierno de Chile, quien no tuvo injerencia alguna en los resultados de la investigación. 


\section{Introducción}

La teoría de la fusión de identidad completa más de una década desde que se publicó el primer artículo que la introdujo al campo de la psicología (Swann et al., 2009). En particular, esta teoría nació con la intención de explicar por qué algunas personas son capaces de realizar sacrificios extremos, al punto de dar la vida por su grupo. Autores como Swann et al. (2009), por ejemplo, plantean que la disposición al sacrificio extremo estaría motivada por una identidad personal y grupal altamente saliente entre los individuos, donde se presenta un sentimiento visceral de unión entre el yo personal y el yo social, de modo que la delimitación entre ambas identidades se hace poco distinguible (véase, p. ej., Gómez \& Vázquez, 2015; Swann et al., 2012).

Como constructo, la fusión de identidad se asocia a los lazos relacionales - los sentimientos hacia los miembros individuales del grupo - y los lazos colectivos - los sentimientos hacia el grupo en su conjunto- - (véase, p. ej., Gómez et al., 2019; Gómez, Brooks et al., 2011), con los cuales se crea una percepción de conexión y fuerza recíproca entre la identidad personal y la identidad grupal (Besta, 2018; Gómez, Brooks et al., 2011). Debido a esta fuerte unidad con el grupo, las personas fusionadas representan a los demás miembros como si fueran sus propios familiares (Robert et al., 2019; Swann, Buhrmester et al., 2014; Whitehouse et al., 2014), y este sentimiento de estrecha unión sería uno de los principales motivadores del comportamiento extremo en favor del grupo (véase, p. ej., Buhrmester \& Swann, 2015; Fredman et al., 2015; Swann \& Buhrmester, 2015).

Respecto a sus bases teóricas, la perspectiva de la fusión de identidad se ha basado en cuatro principios que capturan conceptualmente su naturaleza, a saber: el yo personal agente, la sinergia de la identidad, los lazos relacionales y la irrevocabilidad (Gómez \& Vázquez, 2015; Swann et al., 2012); principios que cuentan con evidencia consistente en múltiples investigaciones, por lo cual se puede afirmar que la base teórica de la fusión de identidad tiene un amplio sustento empírico (véase, p. ej., Besta, Mattingly et al., 2015; Gómez, Morales et al., 2011; Heger \& Gaertner, 2018; Vázquez et al., 2017).

En particular, el principio del yo personal agente sugiere que la persona fusionada mantiene acciones intencionadas que van en beneficio del grupo (Fredman et al., 2015). Estas acciones sobresalen en contextos grupales, donde el yo personal se activa junto con el yo social (Swann, Gómez, Huici et al., 2010). De esta forma, la persona fusionada se siente capaz de realizar comportamientos que, a su juicio, tendrán consecuencias favorables para todo el grupo (Gómez \& Vázquez, 2015).
Por otra parte, el principio de sinergia de la identidad refiere a la sincronía que desarrolla el yo personal con el yo social, con la cual se forma una combinación recíproca entre ambas identidades (Gómez, Brooks et al., 2011; Swann et al., 2009). Esto provoca que cuando se active una identidad, también se active la otra, de modo que el comportamiento de la persona fusionada se amplifica (Heger \& Gaertner, 2018; Swann et al., 2012).

Adicionalmente, el principio de los lazos relacionales refiere a que los sujetos fusionados reconocen a los miembros del grupo tanto por sus identidades personales como por sus identidades sociales (Swann, Gómez, Dovidio et al., 2010). De modo que la combinación de ambas identidades mediante este tipo de reconocimiento desarrolla en las personas fusionadas un fuerte vínculo con los miembros del grupo (véase, p. ej., Besta, 2014; Gómez, Brooks et al., 2011).

Por último, el principio de irrevocabilidad refiere a que las personas fusionadas tienden a permanecer fusionadas a lo largo del tiempo (Vázquez et al., 2017; Swann et al., 2012; Swann et al., 2015), lo cual sucede debido a que estas personas construyen lazos relacionales y colectivos que las vinculan emocionalmente y de manera intensa con el grupo (Gómez et al., 2019). De esta manera, se refuerza el sentido de que su yo social esté unido a su yo personal, generando un sentimiento de gratificación y significado que hace que resulte difícil pensar en la posibilidad de desvincularse del grupo (Gómez, Morales et al., 2011).

Ahora, si bien es posible encontrar algunos estudios teóricos que dan cuenta de las propiedades fundamentales de la teoría de la fusión de identidad (véase, p. ej., Buhrmester \& Swann, 2015; Fredman et al., 2015; Gómez \& Vázquez, 2015; Swann et al., 2012; Swann \& Buhrmester, 2015; Whitehouse \& Lanman, 2014; Yuan et al., 2014), hasta la fecha ningún artículo ha realizado una revisión que sintetice y agrupe de manera sistemática todos los estudios empíricos que han involucrado esta variable. Por lo tanto, el objetivo del presente estudio teórico (Montero \& León, 2007) fue realizar una revisión sistemática de los estudios empíricos (Ato et al., 2013) que han reportado el uso del constructo de la fusión de identidad entre los años 2009 y 2018.

\section{Método}

\section{Procedimiento y estrategias de búsqueda}

Se realizó una revisión sistemática de la literatura siguiendo los lineamientos y recomendaciones de la declaración PRISMA, cumpliendo con los puntos 1-3, 6-11, 14, 17-18, 23-26 y 28 de su lista de comprobación (Liberati et al., 2009; Urrútia \& Bonfill, 2010). Con esto, se configuró 
la exploración de artículos publicados entre los años 2009 y 2018 a partir de la búsqueda en siete bases de datos, de las cuales se obtuvo un total de 351 documentos - Web of Science (87), Scopus (55), ProQuest (65), ScienceDirect (46), Willey Online Library (35), EBSCO (38) y JSTORE (25)-.

En particular, la pesquisa de artículos se limitó a estudios realizados en inglés y español que incluyeran las palabras clave "identity fusion" o "fusión de identidad"; y esta misma se acotó a nueve áreas de investigación: social psychology, social identity, identity, behavior, group dynamics, psychology, group identity, social sciences y social behavior. De los artículos allí encontrados, se incluyeron solo estudios con datos empíricos, y se excluyeron los trabajos teóricos e instrumentales sobre la fusión de identidad.

\section{Selección de los estudios}

Se realizó una selección por etapas (véase Figura 1) en la que, primero, se compilaron todos los artículos recolectados en las bases de datos antes señaladas $(n=351)$; segundo, se leyeron los títulos y se eliminaron los artículos duplicados $(n=142)$; tercero, se realizó la lectura de títulos, resúmenes, palabras clave e instrumentos utilizados, a partir de la cual se eliminaron los artículos que no cumplían con los criterios de inclusión $(n=129)$; y, cuarto, se llevó a cabo una lectura a texto completo, con la cual se procedió a eliminar los estudios teóricos, instrumentales o que no centraban sus resultados en la fusión de identidad $(n=28)$.

\section{Sintesis de resultados}

Una vez finalizada la selección $(n=52)$, se realizó una síntesis de resultados con el fin de comparar los diferentes estudios; procedimiento que se llevó a cabo por medio de la extracción de ocho datos principales: (a) el autor o autores del artículo, (b) el año de publicación, (c) la muestra o muestras con las que se trabajó en el estudio, (d) el diseño de investigación, (e) los instrumentos utilizados para la medida de fusión de identidad, (f) el grupo o grupos de referencia al cual se orienta la fusión de acuerdo a los intereses de cada estudio, (g) las variables o manipulaciones tenidas en cuenta en los estudios, y (h) los principales resultados o hallazgos de cada uno de los artículos.

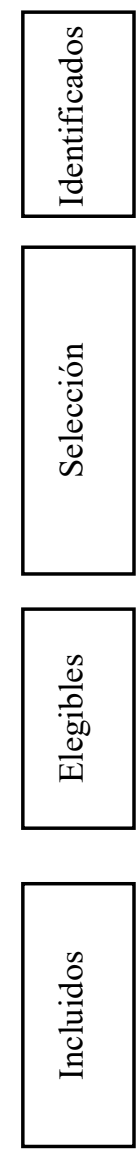
Resultados identificados en las bases de datos $(n=351)$

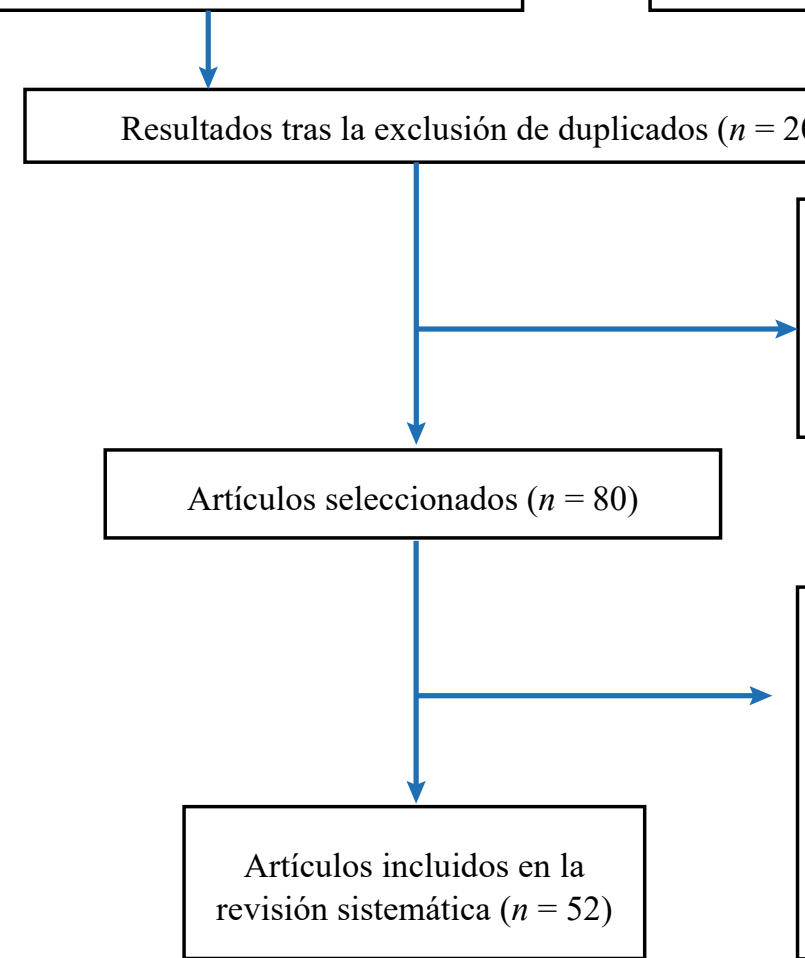

Artículos excluidos en la inspección de títulos, resúmenes, palabras clave, y por no incluir instrumentos de medida de la fusión de identidad $(n=129)$

Figura 1. Diagrama de flujo PRISMA: proceso de identificación y selección de la literatura.

Artículos excluidos por ser estudios teóricos de fusión de identidad $(n=10)$, estudios instrumentales de fusión de identidad $(n=3)$ y estudios que no centraban sus resultados en la fusión de identidad $(n=15)$ 


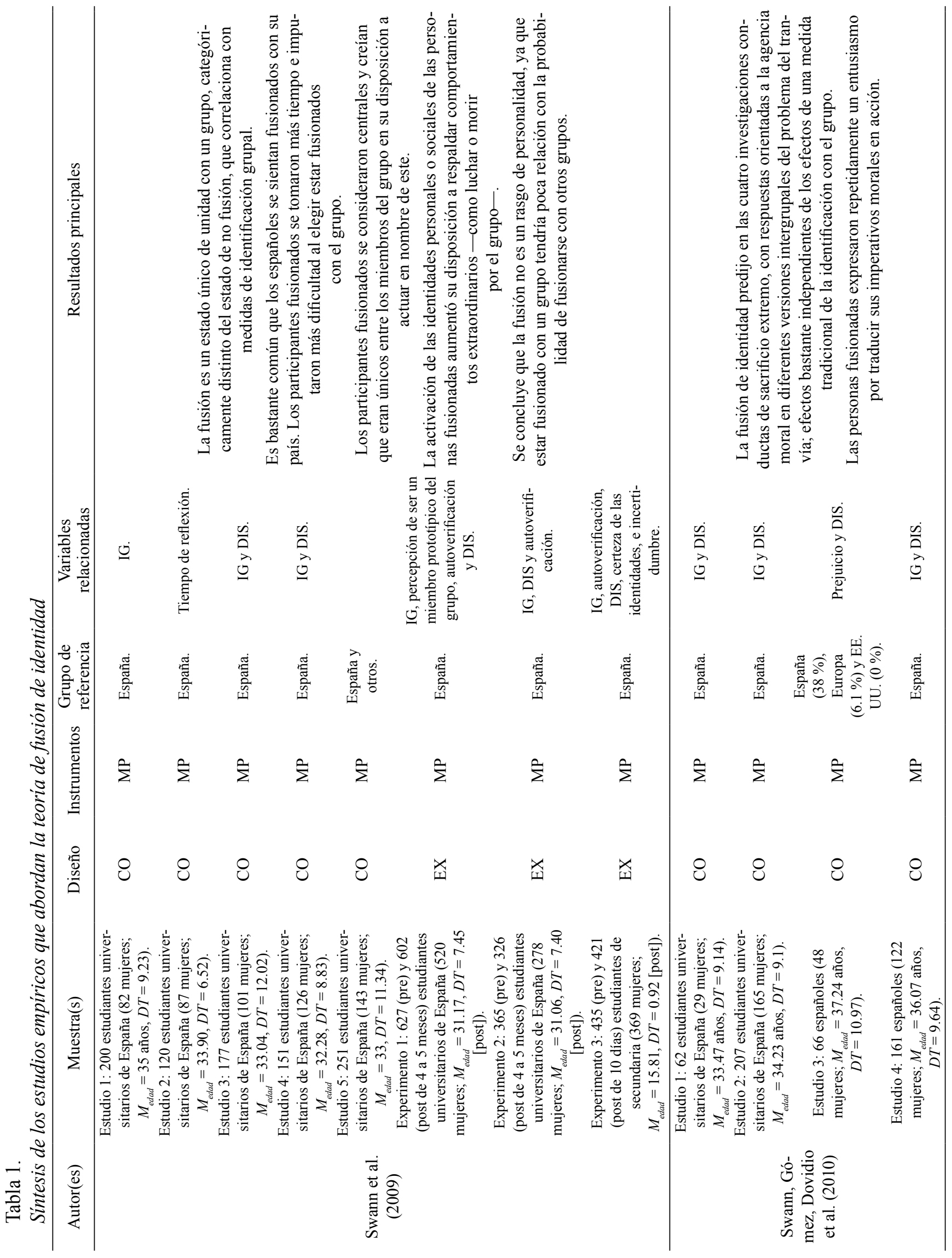




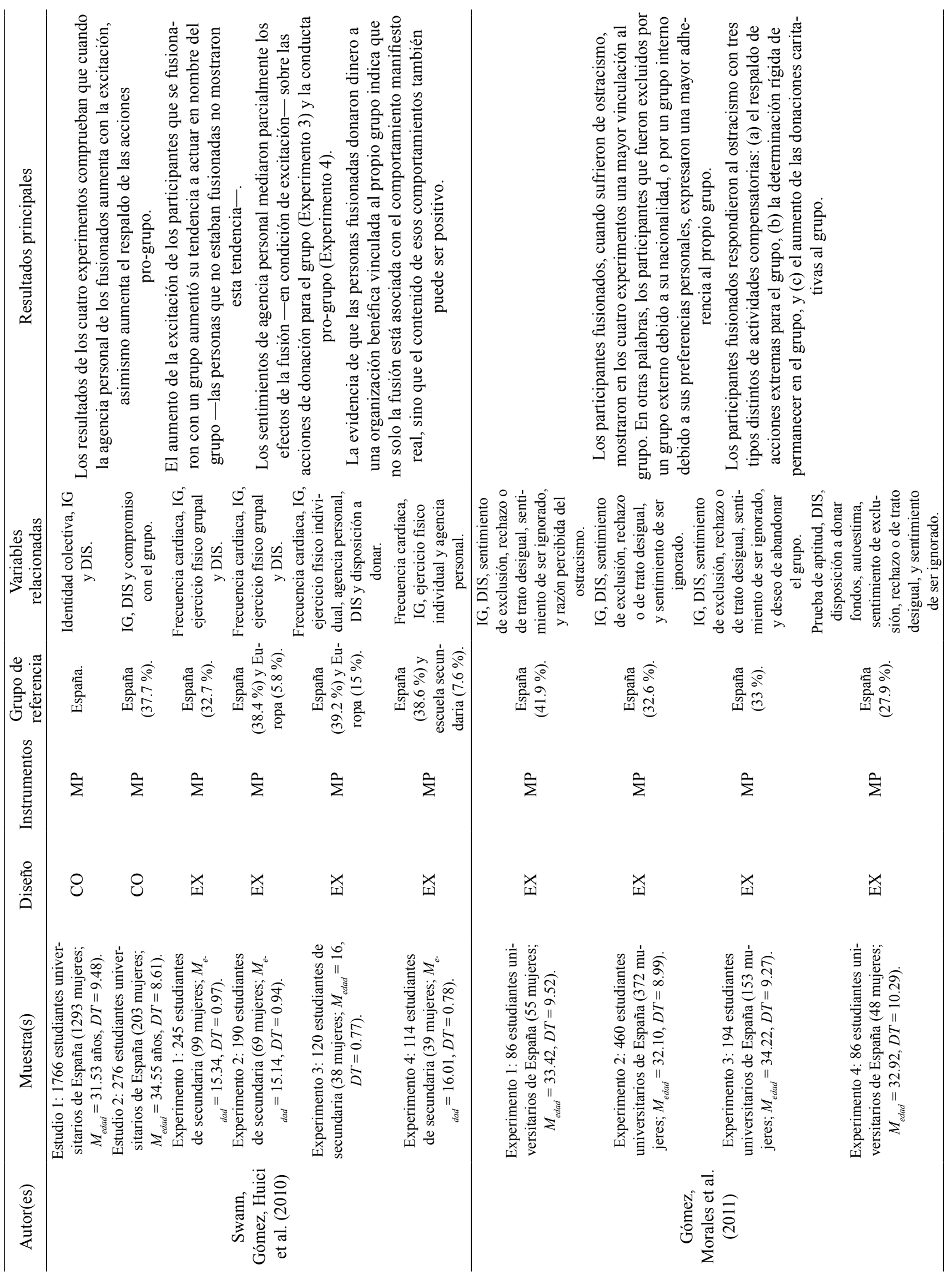




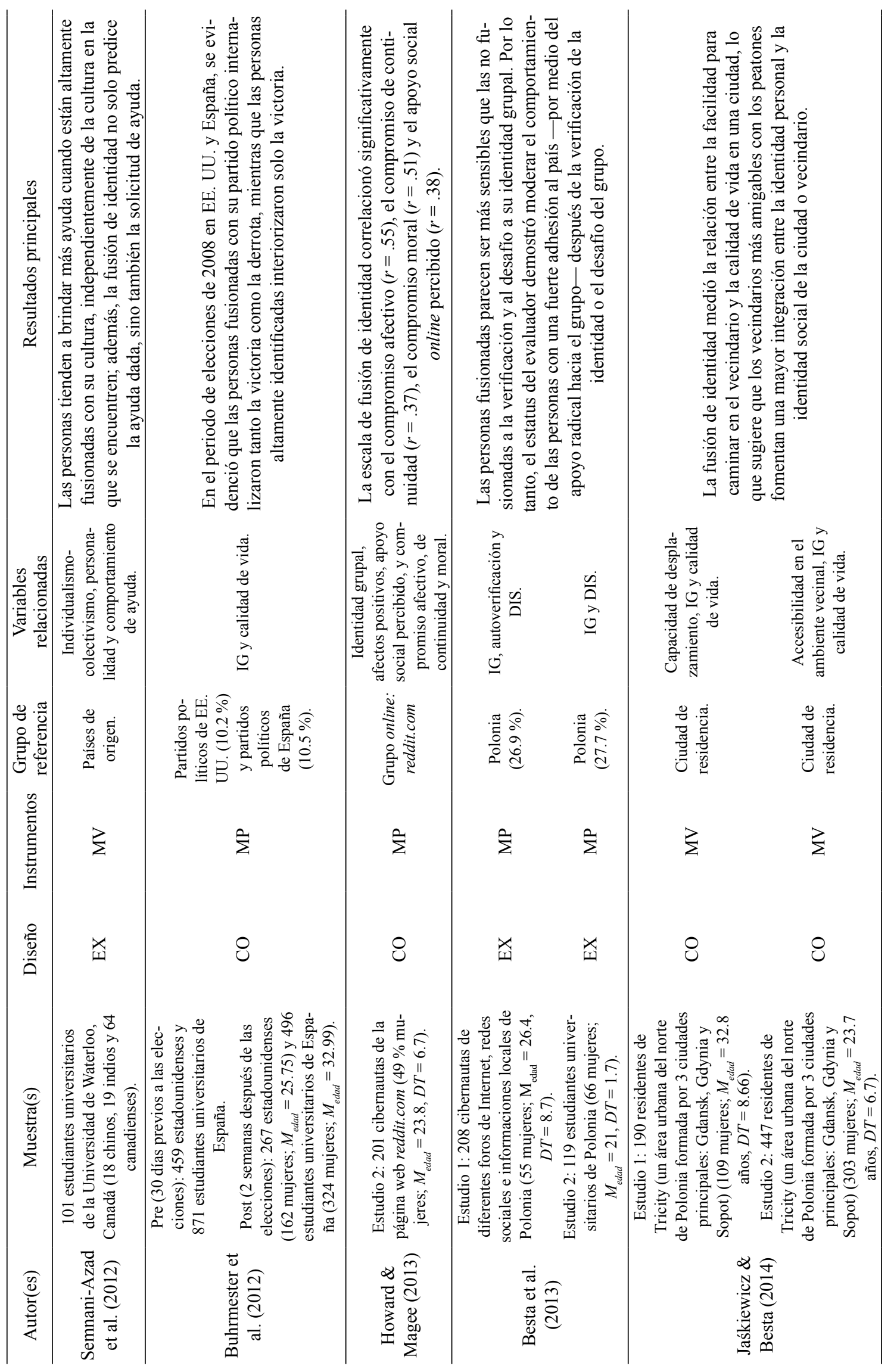




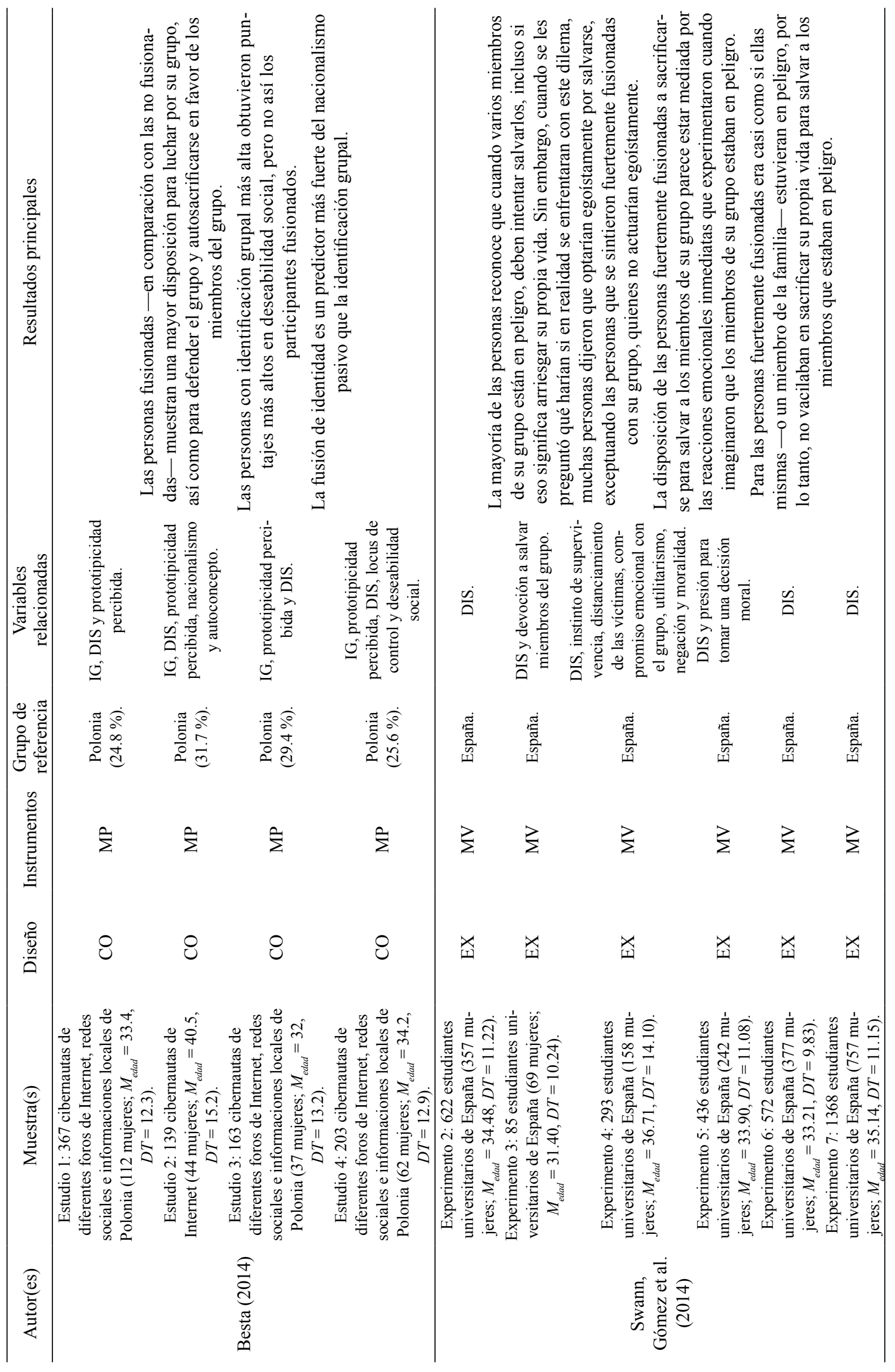




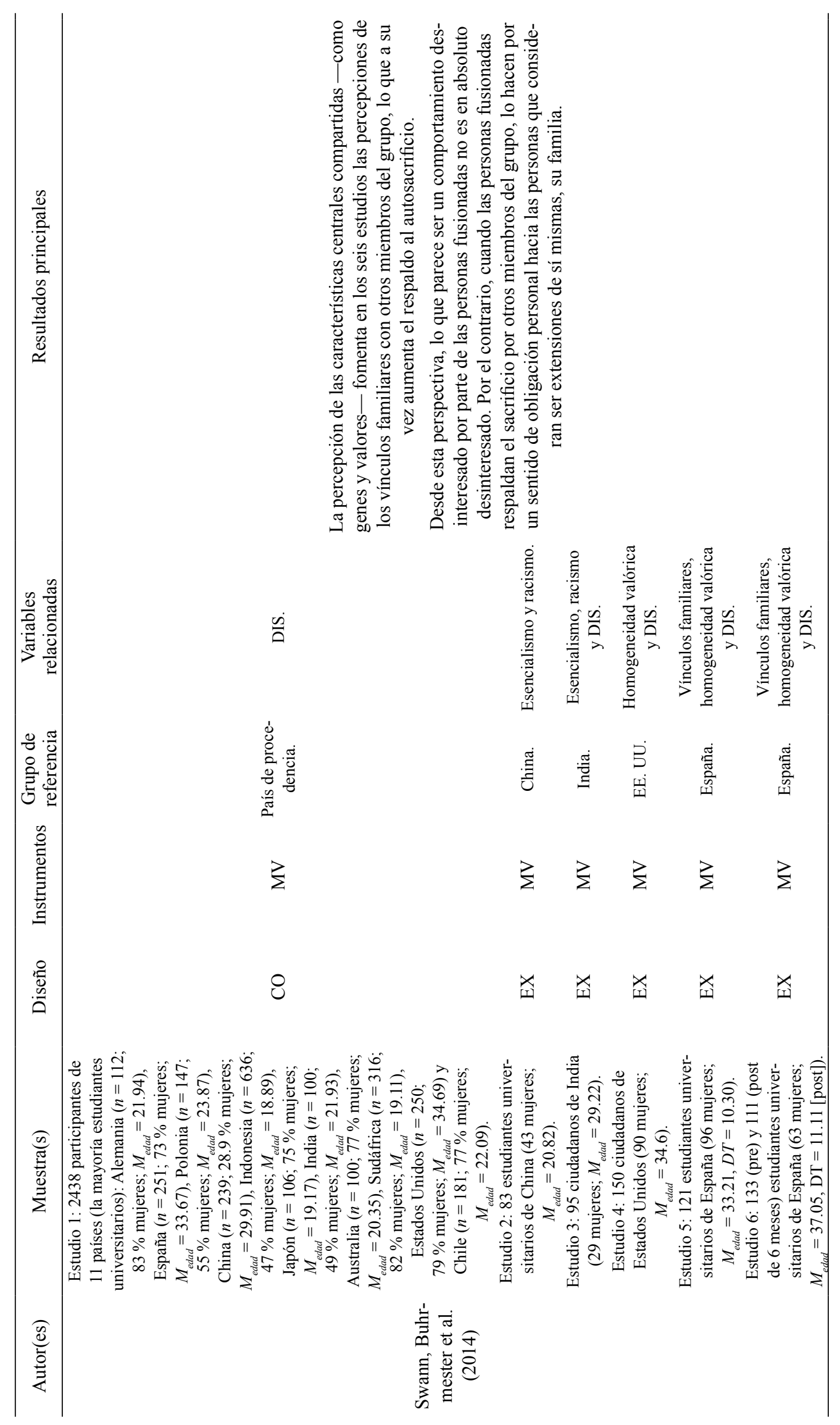




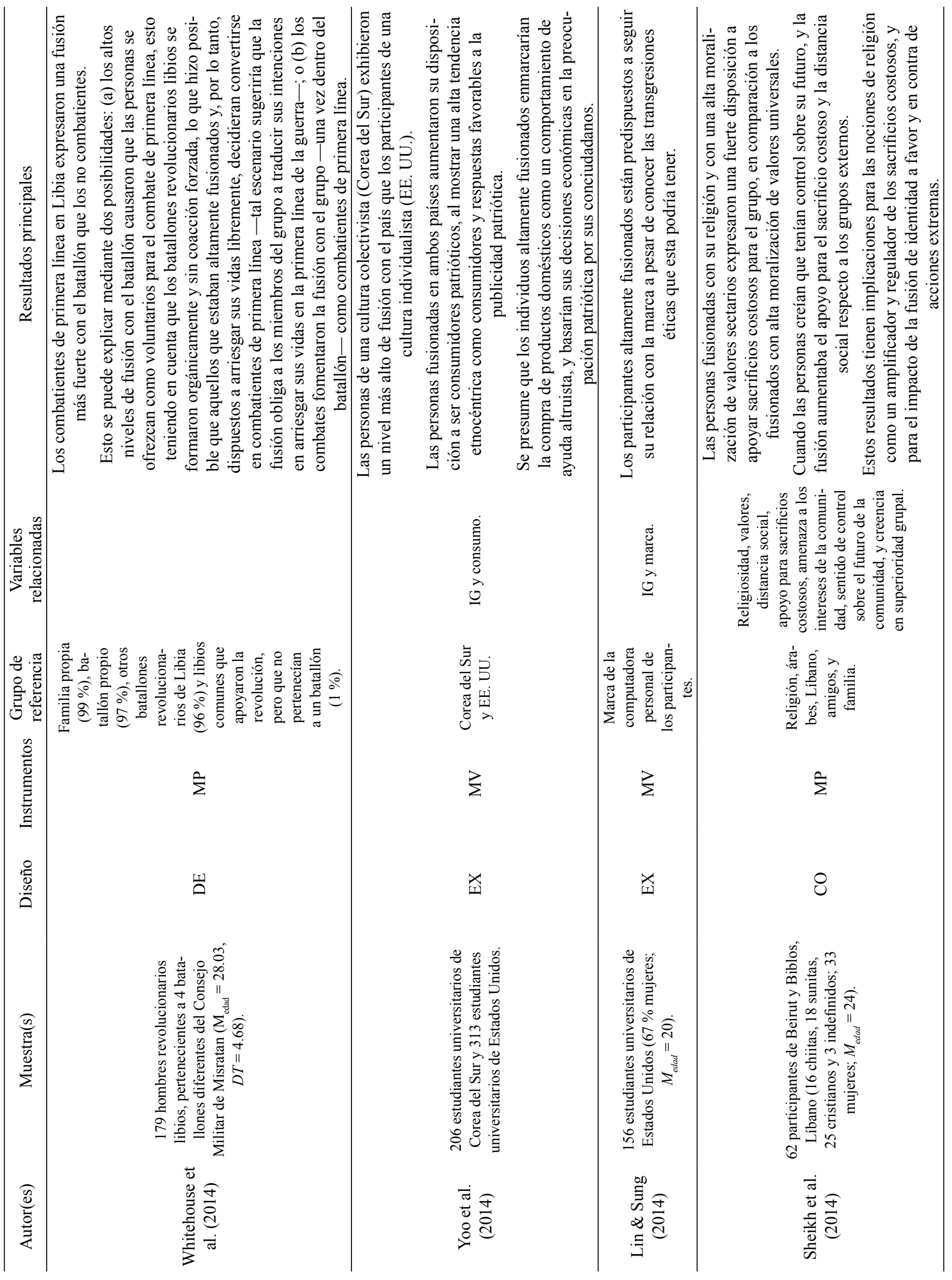




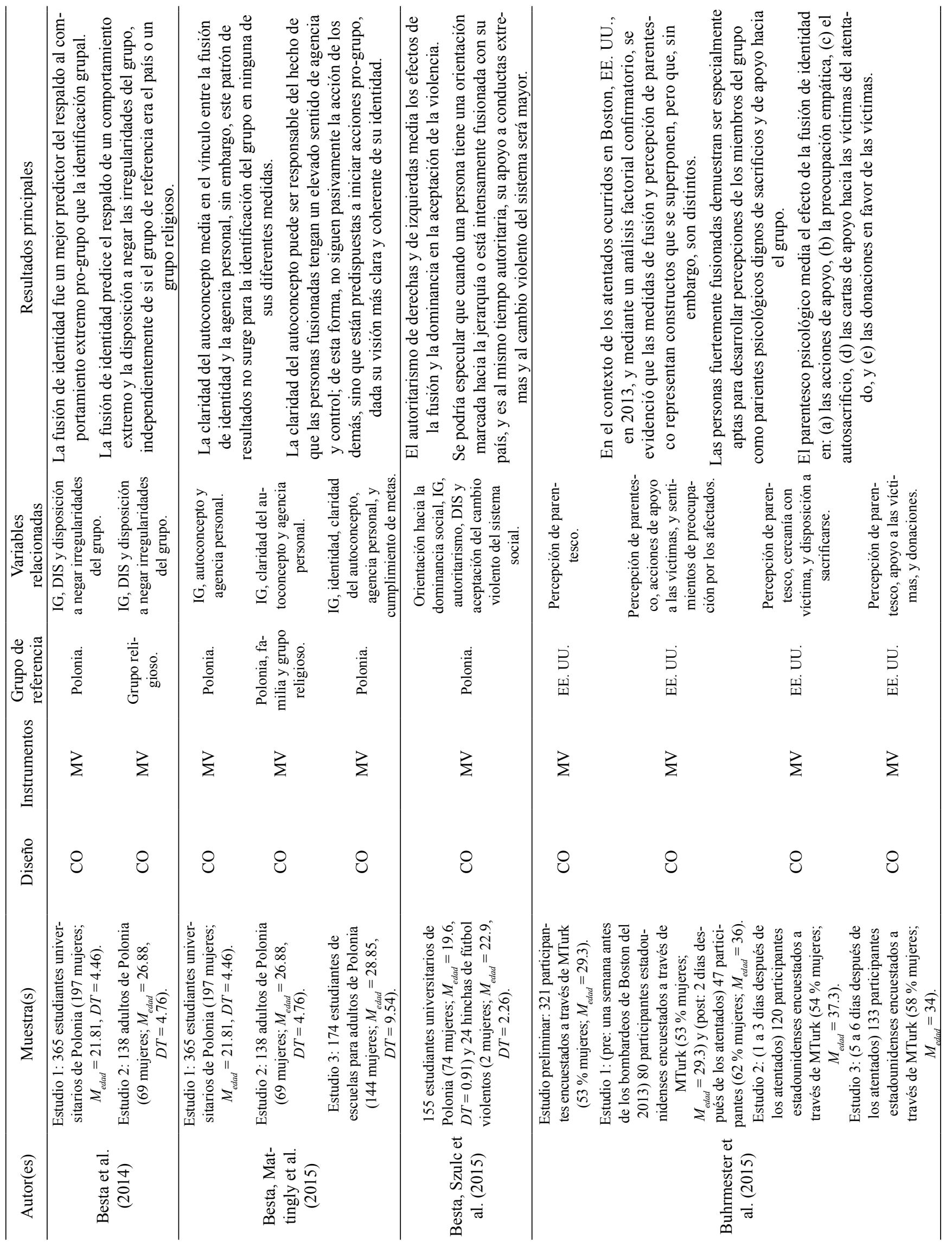




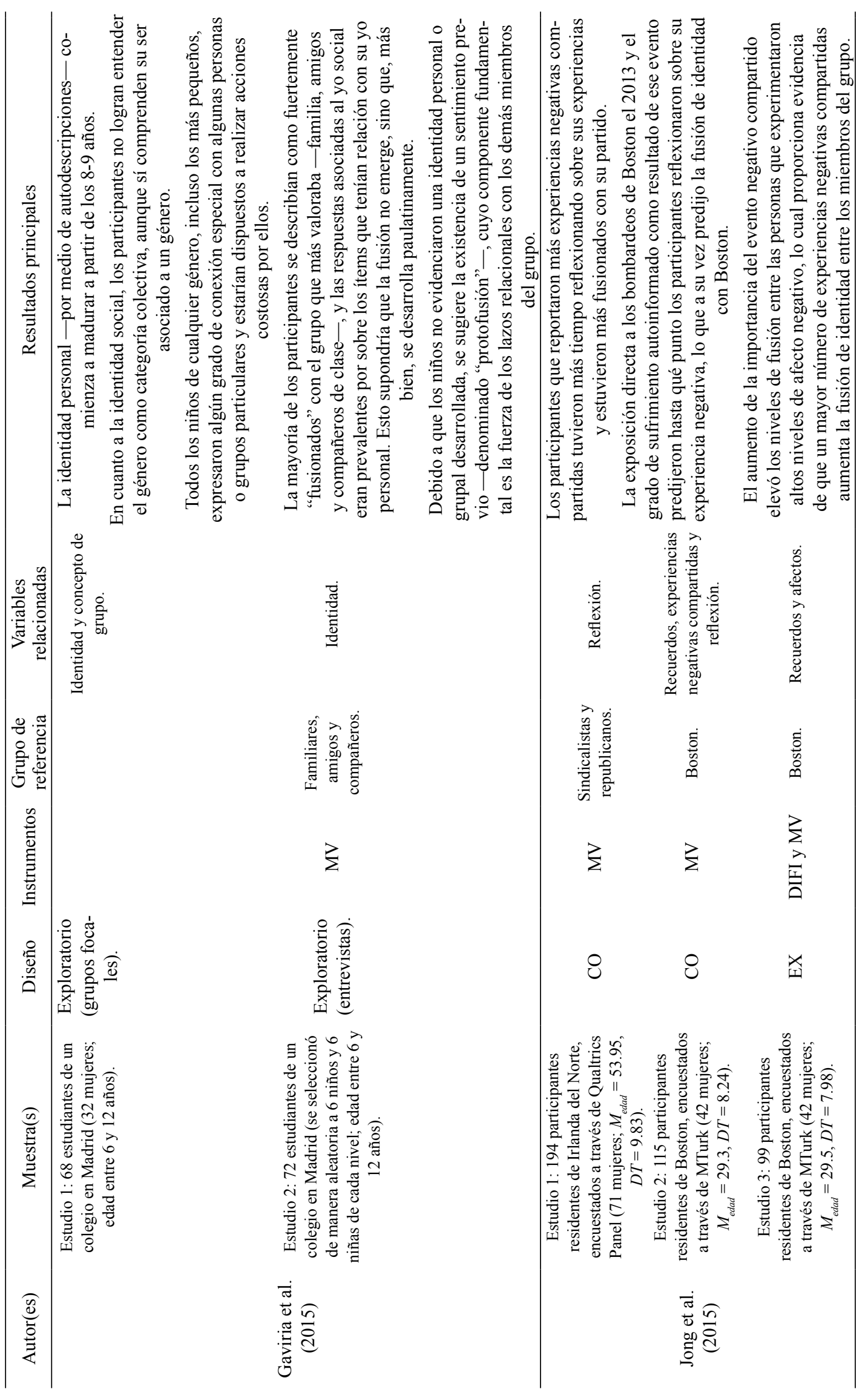




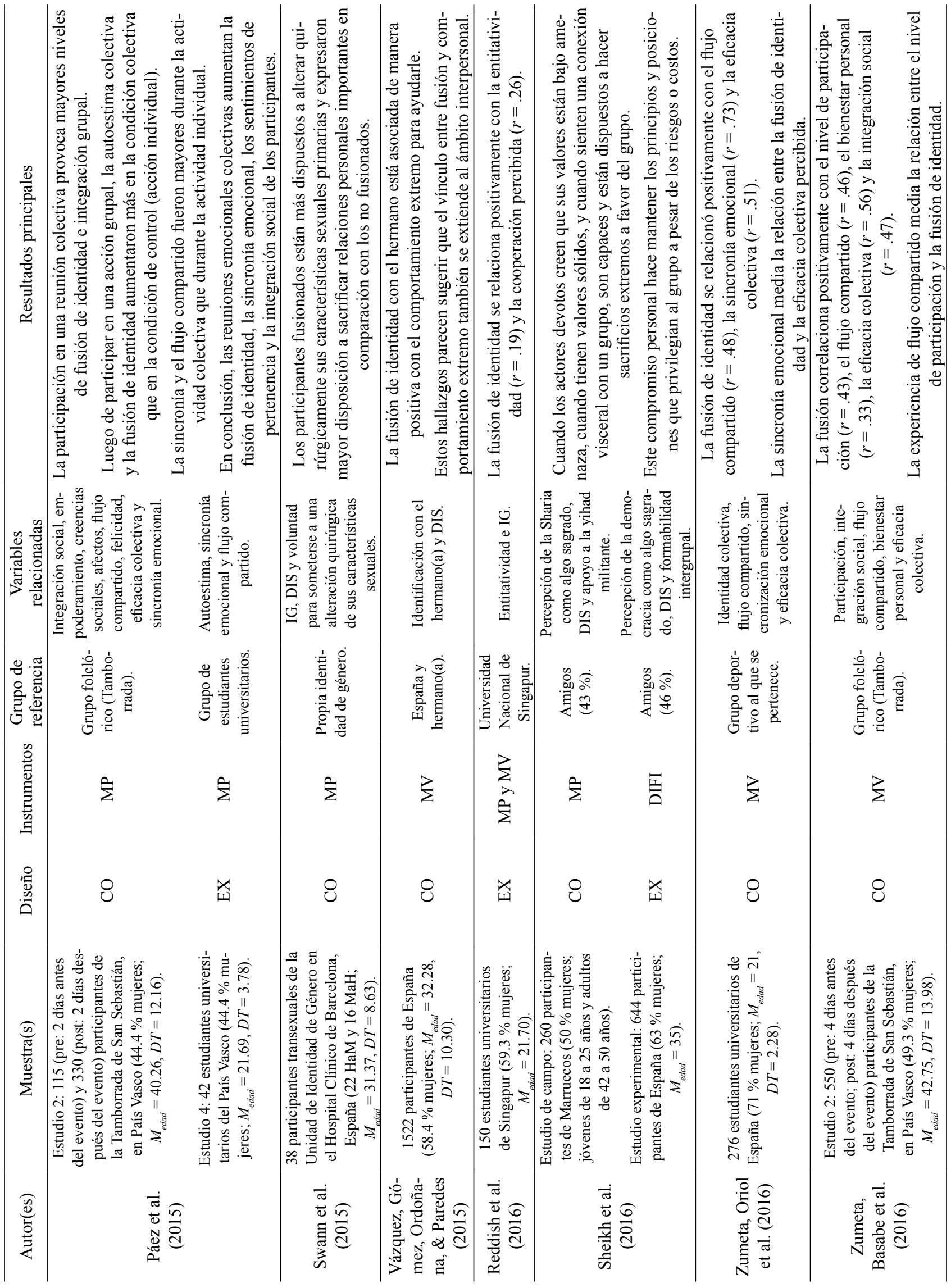




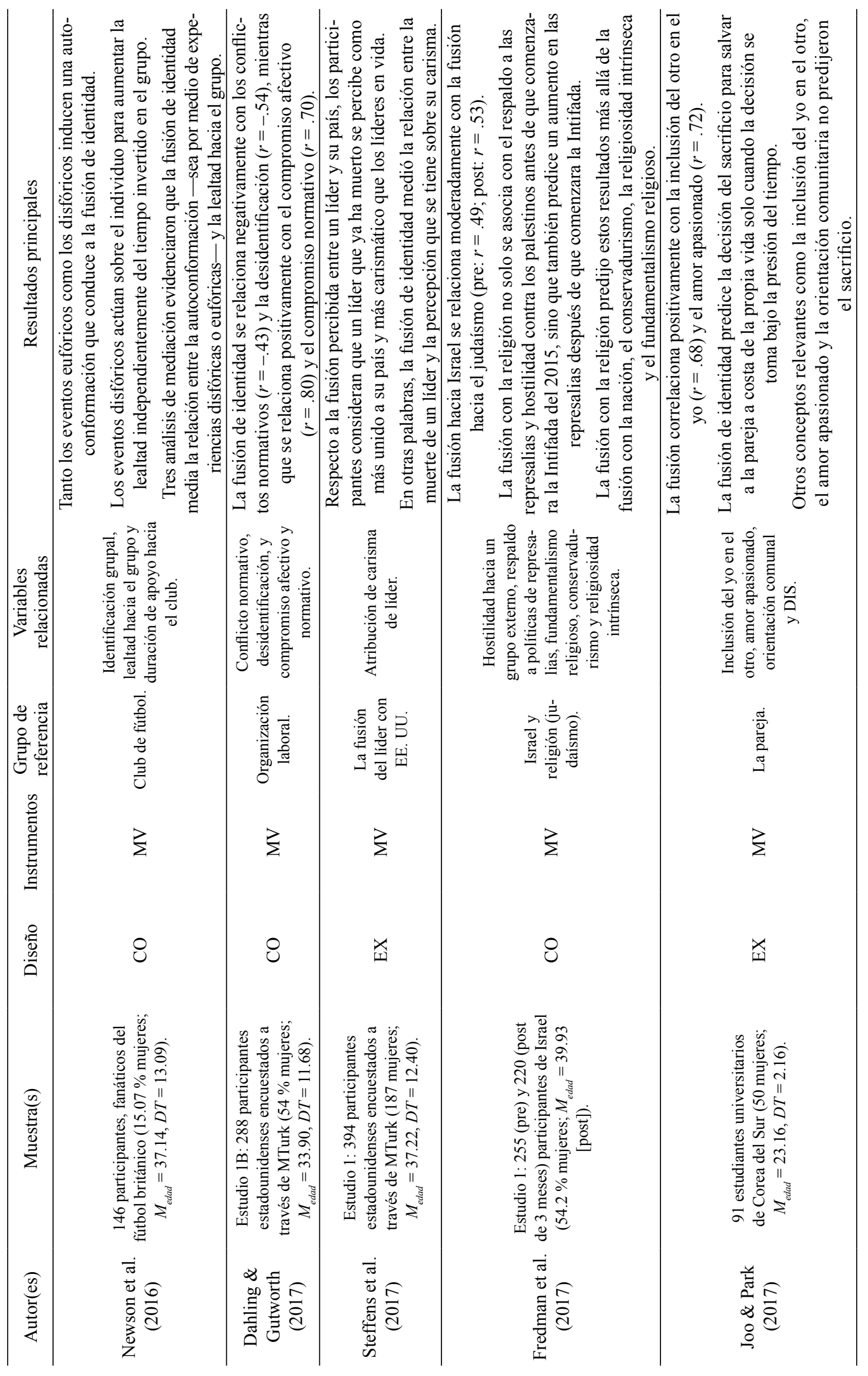




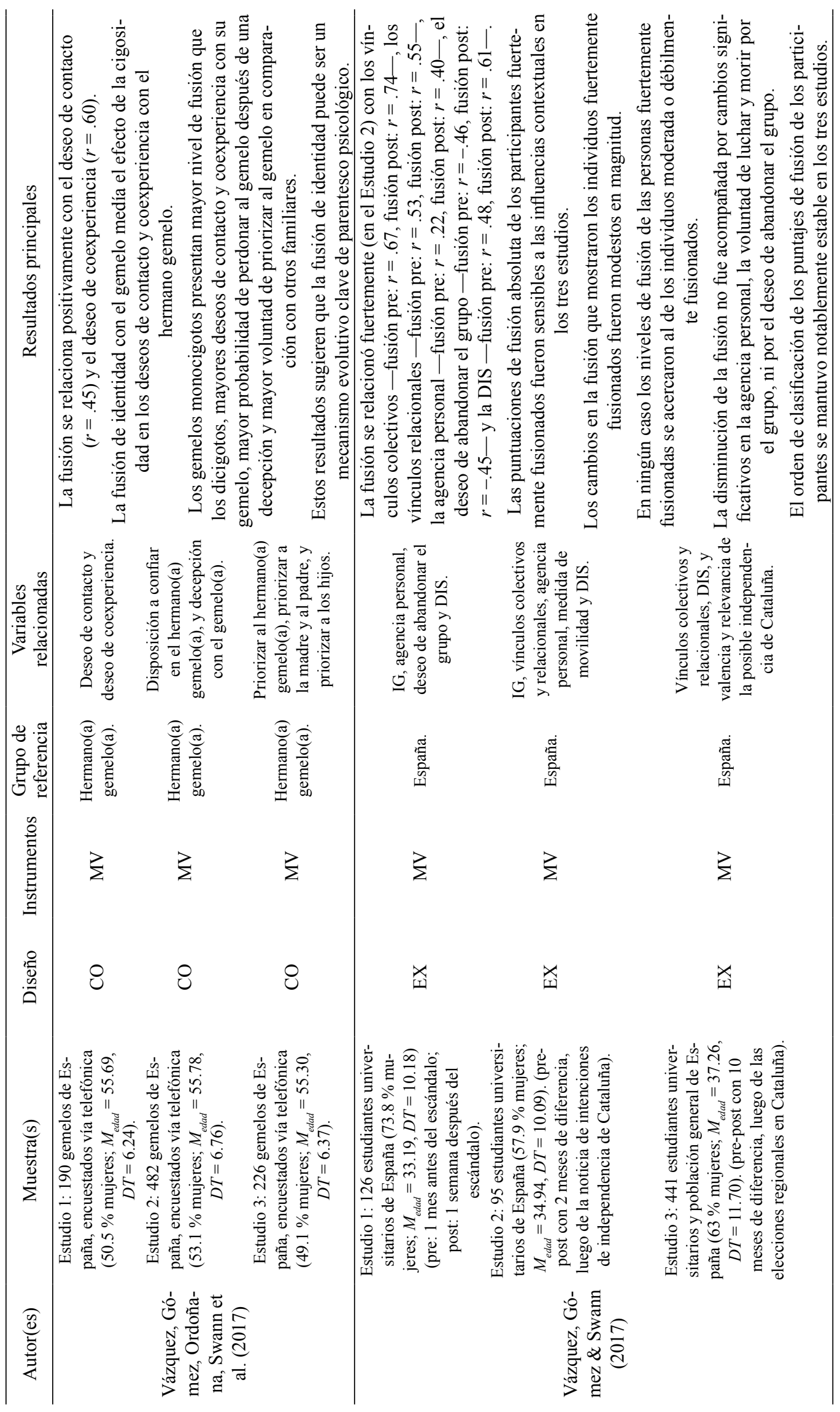




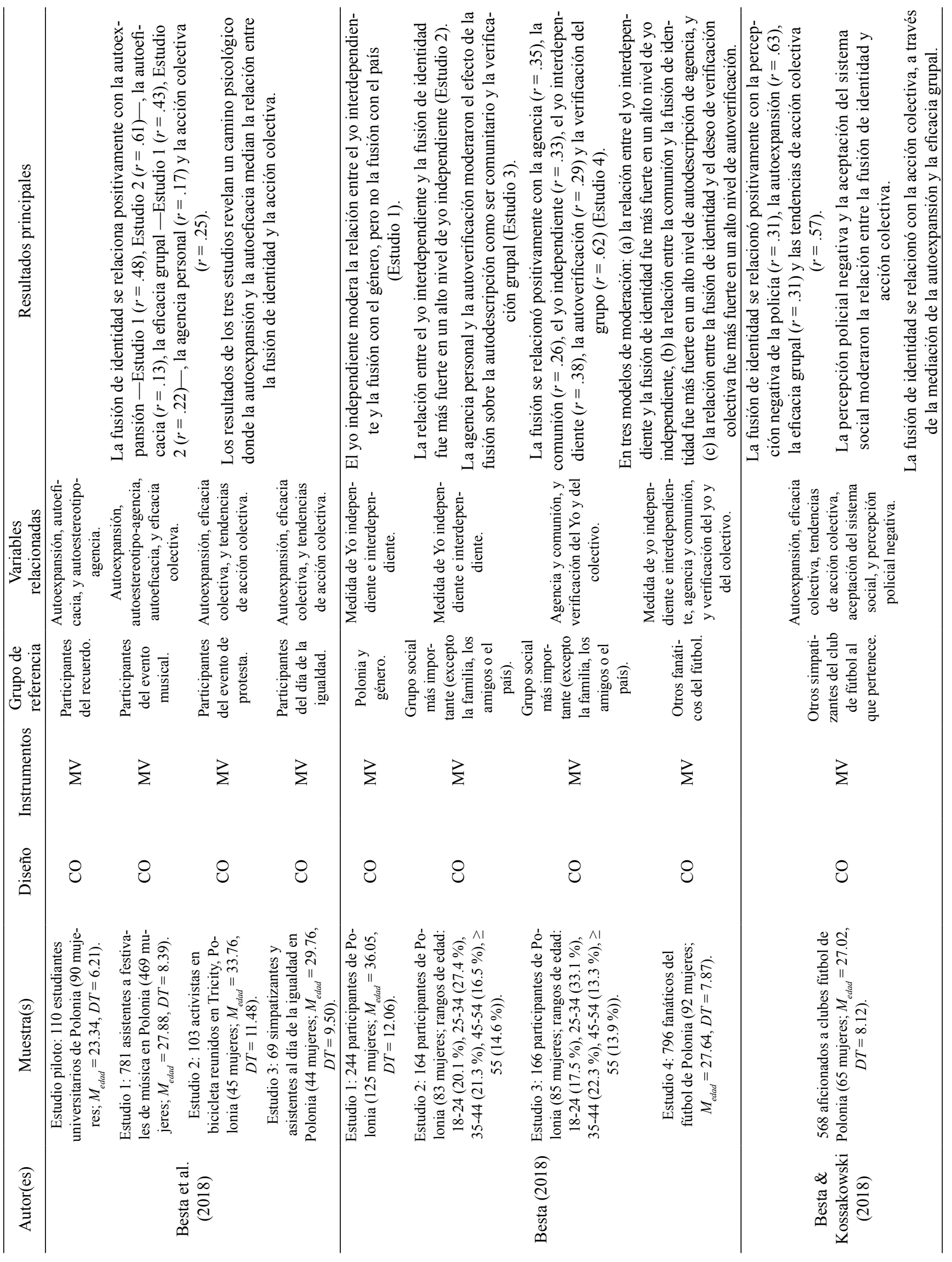




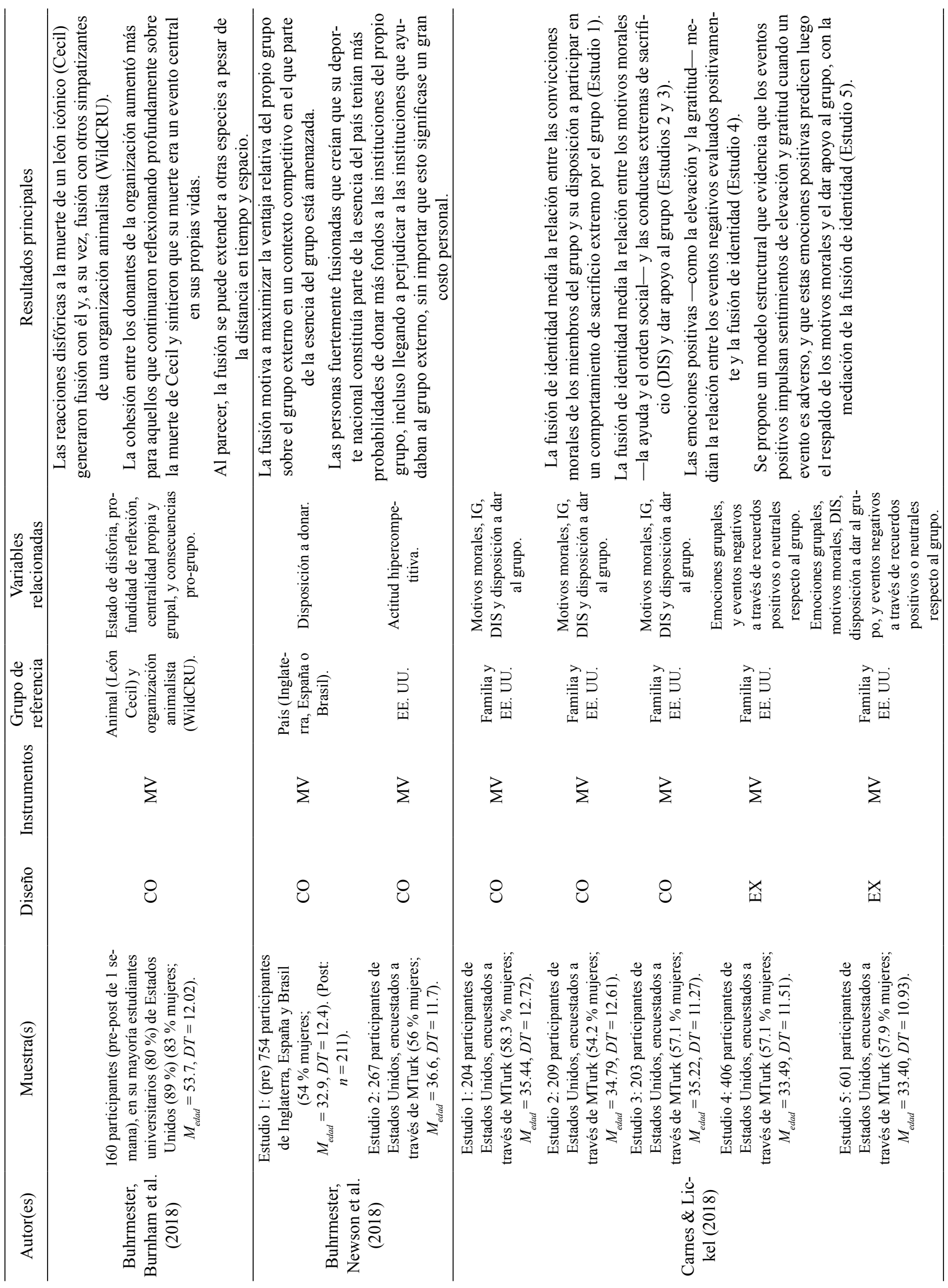


Henríquez, D., Urzúa, A., \& López-López, W.

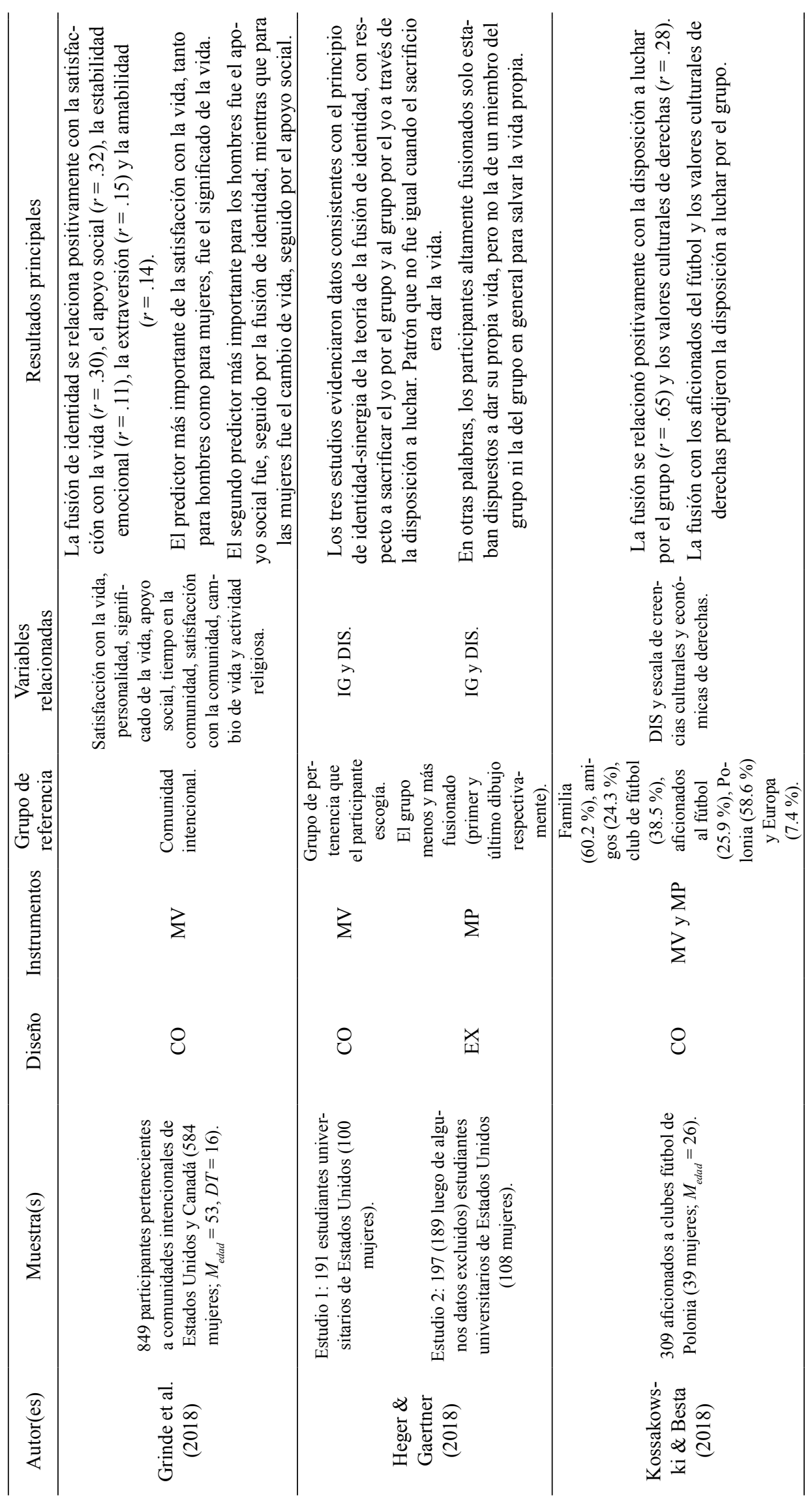




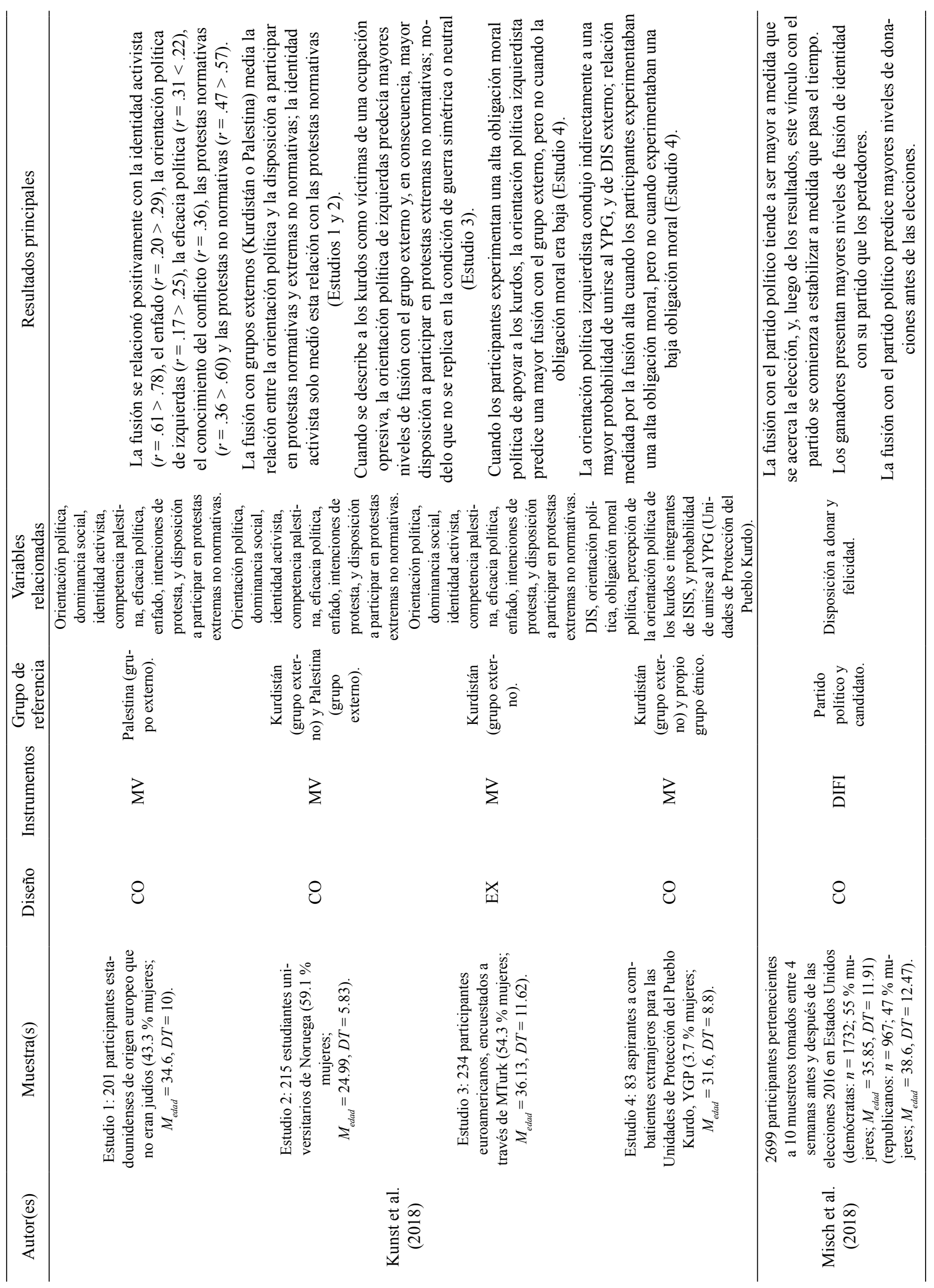




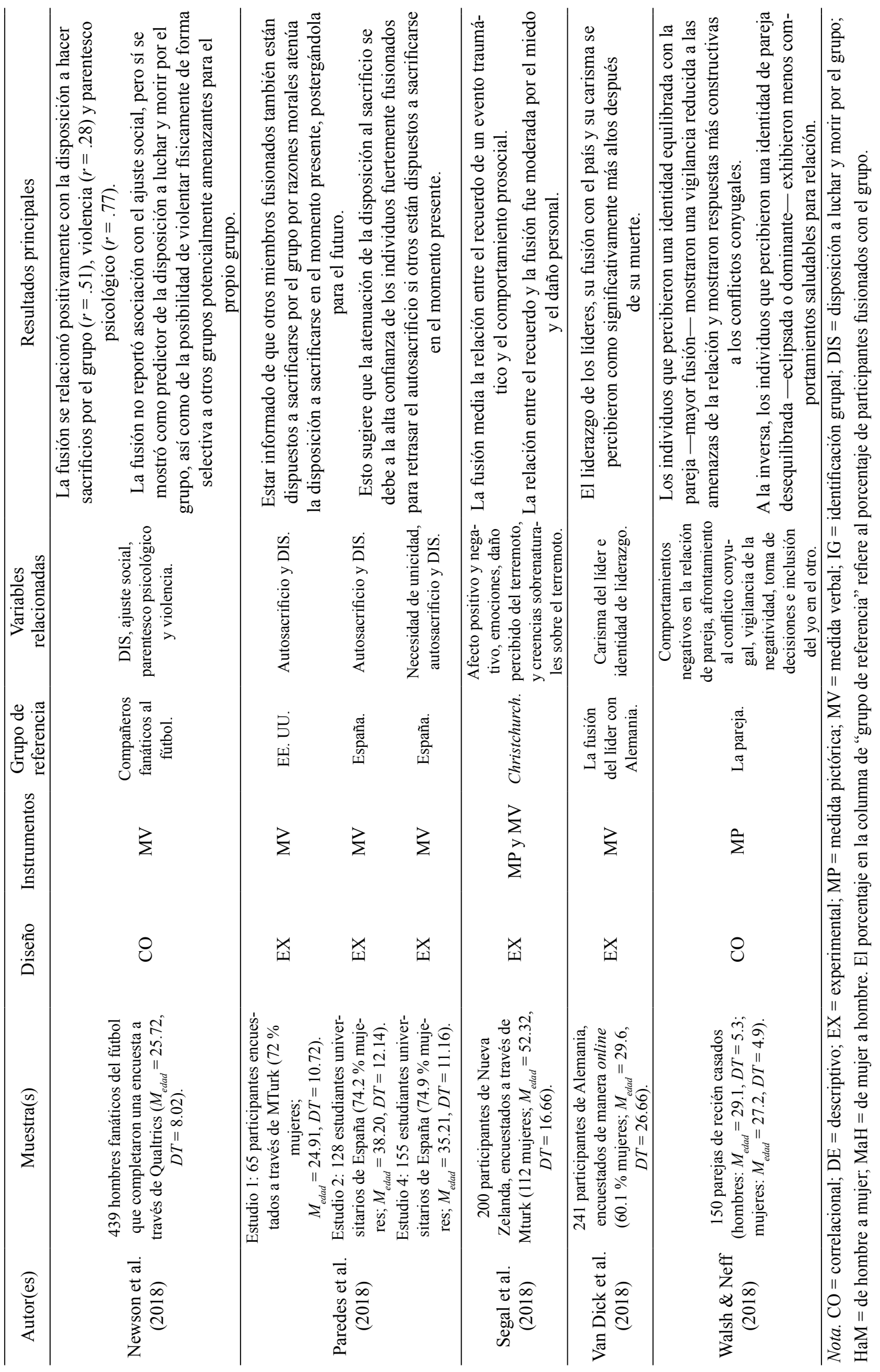




\section{Resultados}

La descripción detallada de las características principales de los 52 artículos incluidos en la revisión sistemática se encuentra en la Tabla 1, sin embargo, resulta importante destacar que, de la totalidad de artículos, se encontraron 117 estudios empíricos que abarcaron la fusión de identidad como parte de sus resultados, y que, respecto a los diseños de investigación de dichos estudios, 69 (59\%) eran correlacionales, 45 (38.5\%), experimentales, dos (1.7\%), exploratorios, y uno $(0.9 \%)$, descriptivo.

Por otra parte, en cuanto a los participantes de los estudios, se encontró que en su totalidad provenían de más de 23 países, siendo los principales España $(38 \% ; n=49)$, Estados Unidos (20.2\%; $n=26)$, Polonia $(18.6 \% ; n=24)$, y otros - donde se agrupan Alemania, Australia, Brasil, Chile, China, Corea del Sur, India, Indonesia, Inglaterra, Irlanda del Norte, Israel, Japón, Líbano, Libia, Marruecos, Noruega, Nueva Zelanda, Singapur y Sudáfrica, entre otros- $(23.3 \% ; n=30)$; y que el $45 \%(n=58)$ correspondía a estudiantes universitarios, el $38.8 \%(n=50)$ a población general, y el $16.3 \%(n=21)$ a otros tipos de población — como activistas, fanáticos del fútbol, gemelos, parejas, guerrilleros, militares, transexuales, niños, niñas y adolescentes-.

Respecto a los instrumentos de medida de la fusión de identidad, el $34.2 \%(n=41)$ de los estudios utilizó una medida pictórica - MP - (Swann et al., 2009), el 63.3\% $(n=76)$ hizo uso de una medida verbal - MV- (Gómez, Brooks et al., 2011), y el $2.5 \%(n=3)$ reportó el uso del indice dinámico de fusión de identidad - DIFI- (Jiménez et al., 2016).
Adicional a esto, en cuanto a los grupos u objetivos de referencia, el $64.1 \%(n=75)$ de los estudios utilizó al país como grupo de referencia, el $14.5 \%(n=17)$ a familiares y amigos, el $29.1 \%(n=34)$ a grupos específicos - como partidos políticos, guerrilleros, militares, grupos deportivos, grupos online, instituciones educativas, comunidades intencionales y ciudades de residencia-, y el $10.3 \%(n=12)$ a otros objetivos de referencia - como candidatos, marcas comerciales y figuras públicas-.

Asimismo, respecto a las variables que más se han estudiado en relación con la fusión de identidad, se encontraron las relacionadas con la identidad $(n=64 ; 54.7 \%)$, la motivación al sacrificio $(n=60 ; 51.3 \%)$, la relación con el grupo $(n=41 ; 35.3 \%)$, algunas variables psicológicas individuales $(n=30 ; 25.6 \%)$, los vínculos $(n=24$; $20.5 \%)$, y otras variables $(n=41 ; 35.3 \%)$; mientras que las variables menos estudiadas fueron los sentimientos y emociones $(n=20 ; 17.1 \%)$, la agencia personal $(n=14$; $12 \%)$, el bienestar $(n=7 ; 6 \%)$, y los afectos $(n=5 ; 4.3 \%)$. La agrupación de las variables estudiadas junto con la fusión de identidad se puede observar en la clasificación que aparece en la Tabla 2.

Por último, en cuanto a los resultados más importantes de los artículos analizados, es de destacar que:

a. la fusión de identidad (FI) es un fuerte predictor de conductas extremas de sacrificio por el grupo (p. ej., Besta et al., 2013; Gómez, Morales et al., 2011; Heger \& Gaertner, 2018; Kunst et al., 2018; Paredes et al., 2018; Sheikh et al., 2016; Swann et al., 2009; Swann et al., 2010; Swann, Buhrmester et al, 2014; Swann, Gómez et al., 2014; Vázquez et al., 2017), así como de conductas que implican

Tabla 2.

Agrupación de variables estudiadas en relación con la fusión de identidad

\begin{tabular}{ll}
\hline \multicolumn{1}{c}{ Categoría } & \multicolumn{1}{c}{ Variables } \\
\hline & Identidad grupal. \\
& Identidad colectiva. \\
& Identidad. \\
& Esencialismo. \\
& Valores (individualismo-colectivismo, homogeneidad valórica, fundamentalismo, \\
Relacionadas con la identidad & conservadurismo, utilitarismo y comunalidad). \\
& Nacionalismo. \\
& Certeza de la identidad. \\
& Identidad activista. \\
& Atribución de carisma del líder. \\
& Disposición a sacrificarse por el grupo. \\
Motivación al sacrificio & Disposición a sacrificarse. \\
& Autosacrificio. \\
& Apoyo para sacrificios costosos. \\
& Afectos positivos. \\
Afectos & Afectos negativos. \\
& Afectos. \\
& Compromiso afectivo. \\
\hline
\end{tabular}




\begin{tabular}{|c|c|}
\hline Emoción y experiencia emocional & $\begin{array}{l}\text { Emociones. } \\
\text { Emociones grupales. } \\
\text { Recuerdo de experiencias negativas. } \\
\text { Emociones negativas (sentirse excluido o sentirse ignorado). } \\
\text { Sincronía emocional. } \\
\text { Amor apasionado. } \\
\text { Enfado. } \\
\text { Disforia. }\end{array}$ \\
\hline Relación con el grupo & $\begin{array}{l}\text { Disposición a dar o donar al grupo. } \\
\text { Compromiso con el grupo y lealtad. } \\
\text { Apoyo al grupo y duración del apoyo. } \\
\text { Preocupación por los miembros del grupo. } \\
\text { Conductas de ayuda a los miembros del grupo. } \\
\text { Disposición a negar irregularidades del grupo y a aceptar la violencia o las conductas } \\
\text { extremas como método de cambio social. } \\
\text { Percepción negativa de un grupo y prejuicio de racismo o androginia. } \\
\text { Deseo de abandonar o integrarse al grupo. } \\
\text { Percepción de cercanía o lejanía con el grupo. } \\
\text { Percepción del grupo como algo sagrado. } \\
\text { Acción colectiva. } \\
\text { Hostilidad hacia un grupo. } \\
\text { Percepción de superioridad del grupo. } \\
\text { Percepción de amenaza hacia el grupo. } \\
\text { Percepción de ser un miembro prototípico del grupo. }\end{array}$ \\
\hline Variables psicológicas individuales & $\begin{array}{l}\text { Autoconcepto. } \\
\text { Locus de control. } \\
\text { Deseabilidad social. } \\
\text { Autoestima. } \\
\text { Autoritarismo. } \\
\text { Autoeficacia. } \\
\text { Personalidad. } \\
\text { Sentido de vida. } \\
\text { Control sobre el futuro. } \\
\text { Capacidad de reflexión. } \\
\text { Ostracismo. } \\
\text { Incertidumbre. } \\
\text { Actitud competitiva. } \\
\text { Necesidad de unicidad, independencia e inclusión con el otro. } \\
\text { Proceso de toma de decisiones. }\end{array}$ \\
\hline Bienestar & $\begin{array}{l}\text { Calidad de vida. } \\
\text { Satisfacción con la vida. } \\
\text { Felicidad. } \\
\text { Bienestar. } \\
\text { Satisfacción con la comunidad. }\end{array}$ \\
\hline Agencia y crecimiento personal & $\begin{array}{l}\text { Agencia personal. } \\
\text { Autoestereotipo agencial. } \\
\text { Empoderamiento. } \\
\text { Autoexpansión. } \\
\text { Flujo. }\end{array}$ \\
\hline Vínculos & $\begin{array}{l}\text { Vínculos familiares (parentesco, identificación, confianza, conductas, priorización y } \\
\text { estilos de afrontamiento). } \\
\text { Vínculos colectivos (apoyo social, participación, deseo de contacto, integración } \\
\text { social, eficacia colectiva, normas y desajuste social). }\end{array}$ \\
\hline Otras & $\begin{array}{l}\text { Moralidad. } \\
\text { Presión para tomar una decisión moral. } \\
\text { Religiosidad. } \\
\text { Consumo de marca. } \\
\text { Instinto de supervivencia. } \\
\text { Cumplimiento de metas, movilidad social, cambio de vida. } \\
\text { Creencias sociales-orientación política. } \\
\text { Aceptación del sistema. } \\
\text { Entitatividad. } \\
\text { Deseo de experiencias. } \\
\text { Ejercicio físico grupal e individual. } \\
\text { Frecuencia cardiaca. }\end{array}$ \\
\hline
\end{tabular}

Nota. Algunas variables podrían pertenecer a más de una categoría. 
otorgar (p. ej., Gómez, Morales et al., 2011; Segal et al., 2018; Semnani-Azad et al., 2012) y pedir ayuda al grupo (p. ej., Reddish et al., 2016; Semnani-Azad et al., 2012);

b. la FI en contextos comerciales puede predecir las preferencias de consumo de productos nacionales (Yoo et al., 2014), sumado a que los consumidores fusionados con sus marcas comerciales están predispuestos a seguir su relación con estas a pesar de tener conocimiento de algunas de sus transgresiones éticas (Lin \& Sung, 2014);

c. la FI se ha relacionado principalmente a conductas extremas de sacrificio por el grupo (p. ej., Besta et al., 2014; Besta, 2014; Besta, Mattingly et al., 2015; Besta, Szulc et al., 2015; Buhrmester et al., 2015; Carnes \& Lickel, 2018; Fredman et al., 2017; Kossakowski \& Besta, 2018; Newson et al., 2018; Sheikh et al., 2016; Swann et al., 2009; Swann et al., 2015; Swann, Buhrmester et al, 2014; Swann, Gómez, Dovidio et al., 2010; Swann, Gómez, Huici et al., 2010; Vázquez et al., 2015; Vázquez, Gómez \& Swann, 2017) o por personas individuales (Joo \& Park, 2017; Vázquez, Gómez, Ordoñana et al., 2017; Walsh \& Neff, 2018);

d. la FI se investiga en relación con la identidad grupal (p. ej., Besta et al., 2014; Besta, Mattingly et al., 2015; Besta, Szulc et al., 2015; Buhrmester et al., 2012; Swann et al., 2009; Swann, Gómez, Huici et al., 2010), el apoyo y compromiso grupal (p. ej., Besta, 2018; Besta \& Kossakowski, 2018; Besta et al., 2018; Buhrmester et al., 2015; Buhrmester, Newson et al., 2018; Carnes \& Lickel, 2018; Dahling \& Gutworth, 2017; Howard \& Magee, 2013; Misch et al., 2018; Sheikh et al., 2014; Swann, Gómez et al., 2014; Vázquez, Gómez, \& Swann, 2017; Zumeta, Basabe et al., 2016), el apoyo social percibido (p. ej., Grinde et al., 2018; Howard \& Magee, 2013), la lealtad (Newson et al., 2016) -incluso en condiciones de ostracismo (Gómez, Morales et al., 2011) - , los sentimientos, afectos y emociones hacia el grupo (Buhrmester, Burnham et al., 2018; Carnes \& Lickel, 2018; Dahling \& Gutworth, 2017; Grinde et al., 2018; Howard \& Magee, 2013; Jong et al., 2015; Joo \& Park, 2017; Páez et al., 2015; Segal et al., 2018; Swann, Gómez et al., 2014; Zumeta, Basabe et al., 2016; Zumeta, Oriol et al., 2016), y los valores morales (Kunst et al., 2018; Sheikh et al., 2014; Swann, Gómez et al., 2014), sagrados (Carnes \& Lickel, 2018; Sheikh et al., 2016), democráticos (Sheikh et al., 2016) y culturales (Kossakowski \& Besta, 2018); y que e. la FI se relaciona con la percepción de parentesco (Buhrmester et al., 2015), la calidad de vida (p. ej., Buhrmester et al., 2012; Jaśkiewicz \& Besta, 2014), la satisfacción con la vida (Grinde et al., 2018), la percepción de carisma de un líder (Steffens et al., 2017; van Dick et al., 2018), la religiosidad (Besta et al., 2014; Fredman et al., 2017; Sheikh et al., 2014), el nacionalismo (Besta, 2014), el extremismo político (Besta, Szulc et al., 2015), la orientación política (Kunst et al., 2018) y el respaldo a irregularidades del grupo (Besta et al., 2014).

Por otra parte, los modelos de mediación dentro de los estudios analizados han demostrado que:

a. la FI cumple una función mediadora en la relación entre la accesibilidad del barrio y la calidad de vida en el vecindario (Jaśkiewicz \& Besta, 2014), las convicciones morales y la disposición a comportamientos de sacrificio extremo (Carnes \& Lickel, 2018), el recuerdo de un evento traumático y el comportamiento pro-social (Segal et al., 2018), la autoconformación y la lealtad hacia el grupo (Newson et al., 2016), y la muerte de un líder y la percepción que se tiene sobre su carisma (Steffens et al., 2017);

b. en tres estudios, la FI con el gemelo medió el efecto que ejercía la cigosidad sobre los deseos de contacto y la coexperiencia con el o la hermana gemela (Vázquez, Gómez, Ordoñana et al., 2017);

c. la FI con un animal icónico - o famoso- recientemente muerto medió la relación entre el estado de disforia y la fusión con una organización animalista (Buhrmester, Burnham et al., 2018);

d. la agencia personal media la relación entre la FI —en condición de excitación-y la acción de donar dinero al grupo (Swann, Gómez, Huici et al., 2010), mientras que la agencia personal y la invulnerabilidad median la relación entre la FI y la disposición a lucha y morir por el grupo (Gómez, Brooks et al., 2011), el compromiso emocional media la relación entre la fusión de identidad y el sacrificio extremo (Swann, Gómez et al., 2014), y la claridad del autoconcepto media la relación entre la FI y la agencia personal (Besta, Mattingly et al., 2015);

e. los autoritarismos de derechas y de izquierdas median la relación entre la fusión de identidad y la inclinación hacia la violencia para el cambio del sistema social (Besta, Szulc et al., 2015), mientras que el autoritarismo de derechas media la relación 
entre la FI y la disposición de luchar y morir por el país (Besta, Szulc et al., 2015);

f. el parentesco psicológico media el efecto de la FI sobre las acciones de apoyo, la preocupación empática y el autosacrificio (Buhrmester et al., 2015);

g. el tiempo de reflexión sobre eventos negativos media la relación entre una experiencia negativa compartida y la FI (Jong et al., 2015);

h. la sincronía emocional media la relación entre el nivel de participación colectiva y la FI (Páez et al., 2015), y también la relación entre la FI y la eficacia colectiva (Zumeta, Oriol et al., 2016);

i. la experiencia de flow compartido media la relación entre el nivel de involucramiento en una actividad colectiva y la FI (Zumeta, Basabe et al., 2016);

j. los vínculos colectivos, pero no relacionales, median la FI antes y después de tres eventos históricos negativos para la estabilidad del grupo (Vázquez, Gómez \& Swann, 2017);

k. la FI con un grupo externo media la relación entre la orientación política de izquierda y la protesta extrema (Kunst et al., 2018);

1. la autoexpansión y la eficacia grupal median la relación entre la FI y la acción colectiva (Besta et al., 2018; Besta \& Kossakowski, 2018); y

m. la identidad del liderazgo y la FI median la relación entre la muerte de un líder y la percepción que se tiene sobre su carisma (van Dick et al., 2018).

De igual forma, en cuanto a los modelos de moderación que han incluido a la FI se ha señalado que: (a) el estatus de un evaluador del grupo modera la relación entre la FI y el apoyo radical hacia el grupo (Besta et al., 2013); (b) los valores moderan la relación entre la FI y realizar sacrificios costosos (Sheikh et al., 2014); (c) los afectos negativos moderan la relación entre el recuerdo de una experiencia negativa y la FI(Jong et al., 2015); (d) la impulsividad modera la relación entre la FI con la pareja y el autosacrificio por la pareja (Joo \& Park, 2017); (f) el yo independiente modera la relación entre el yo interdependiente y la FI (Besta, 2018); (g) la autoverificación modera la relación entre la verificación grupal y la FI (Besta, 2018); (h) la autodescripción como agente modera la relación entre la autodescripción como un ser comunitario y la FI (Besta, 2018); y, finalmente, (i) la percepción policial negativa y la aceptación del sistema moderan la relación entre la FI y la acción colectiva (Besta \& Kossakowski, 2018).

Por último, cabe mencionar que en los modelos de mediación moderada se encuentra, adicionalmente, que: (a) los valores compartidos moderan el efecto de la FI sobre los vínculos familiares, mientras que los vínculos familiares median la relación entre la FI y el respaldo de acciones extremas por el grupo (Swann, Buhrmester et al., 2014); (b) la confianza en el sacrificio de otros fusionados media la relación entre la FI y la disposición a luchar y morir por el grupo, al mismo tiempo que la justificación del sacrificio modera el efecto de la confianza en el sacrificio de otros fusionados y la FI sobre la disposición a luchar y morir por el grupo (Paredes et al., 2018); y, finalmente, (c) el miedo y el daño personal causado por un desastre natural moderan el efecto del recuerdo del desastre natural sobre la FI, mientras que la FI media la relación entre el recuerdo del desastre natural y el comportamiento prosocial (Segal et al., 2018).

\section{Discusión}

El propósito del presente trabajo fue revisar y sintetizar los estudios empíricos que hicieran uso de la teoría de la fusión de identidad entre los años 2009 a 2018. Teniendo esto en cuenta, los resultados principales de los artículos analizados indican que la fusión de identidad es un fuerte predictor de conductas extremas de sacrificio por el grupo. Específicamente, las personas fusionadas estarían altamente orientadas al apoyo y compromiso grupal, por lo que crearían fuertes lazos relacionales a través de sentimientos, afectos y emociones hacia el grupo - involucrando valores morales, considerados sagrados e irrenunciables-, lo cual tiene como consecuencia que las personas fusionadas sientan que los miembros de su grupo son una extensión de su propia familia - a la cual deben proteger con ahínco- A partir de esto, es importante mencionar que las teorías clásicas (p. ej., Tajfel \& Turner, 1979) no consideran la relevancia de los lazos relacionales dentro de sus constructos, por lo cual no logran generar modelos que alcancen a predecir conductas de sacrificio extremo por el grupo; contrario a lo que propone la teoría de la fusión de identidad (Gómez et al., 2019), donde sí se considera la importancia de los vínculos interpersonales de las personas fusionadas y los miembros de su grupo.

Por otra parte, la presente revisión demuestra que el desarrollo sistemático de evidencias que sustentan la teoría de la fusión de identidad va en continuo progreso, pues esta se ha aplicado a diversas poblaciones - como hooligans, fanáticos del fútbol, terroristas, combatientes, hermanos gemelos, parejas románticas, luchadores, militares, activistas, parejas, guerrilleros, transexuales, y niños, niñas y adolescentes-, ha llegado a países de los cinco continentes - donde destacan España, Estados Unidos, Polonia, Alemania, Australia, Brasil, Chile, China, Corea del Sur, India, Indonesia, Inglaterra, Irlanda del Norte, Israel, Japón, Líbano, Libia, Marruecos, Noruega, Nueva 
406

Zelanda, Singapur y Sudáfrica-, y ha teniendo como objeto de referencia a grupos bastante diversos - como países, familiares, amigos, partidos políticos, escuadrones de combate, grupos deportivos, grupos online, instituciones educativas, comunidades intencionales, ciudades de residencia, candidatos, marcas comerciales y figuras públicas-; además de que se ha aplicado para predecir el sacrificio extremo, incluso a través de mediciones de comportamiento real - como donaciones, cambio de sexo, rendimiento en un video-juego o respuestas a dilemas del tranvía, entre otros-.

Sin embargo, a pesar de que toda la evidencia presentada en la revisión es de gran valor investigativo y social, es importante considerar y prestar atención a algunas limitaciones y nuevas directrices que ayudarían a mejorar y amplificar el desarrollo de los estudios sobre la fusión de identidad, como:

a. que la mayoría de los participantes en los estudios de fusión de identidad eran españoles, estadounidenses y polacos, siendo la mitad de ellos estudiantes universitarios, es decir, población WEIRD —entendida como sujetos pertenecientes a países occidentales, educados, industrializados, ricos y democráticos (Western, Educated, Industrialized, Rich and Democratic [WEIRD]) (Henrich et al., 2010)_, lo que representa un problema habitual de las investigaciones en psicología, y por lo cual se hace necesario seguir indagando el comportamiento de la teoría de fusión de identidad en otros contextos socioculturales (Henrich et al., 2010);

b. que gran parte de la evidencia que relaciona la fusión de identidad y el sacrificio extremo por el grupo se basa en la escala de disposición a luchar y morir de Swann et al. (2009), por lo que sería deseable seguir dando evidencia del poder de predicción de la fusión de identidad utilizando diferentes instrumentos — como la escala de sacrificio personal (Bélanger et al., 2014) — o situaciones que midan el sacrificio extremo por el grupo - como la medida implícita "The Boom Task" (Bélanger et al., 2014; Bélanger et al., 2018) -;

c. que la mayor cantidad de estudios utiliza como grupo de referencia el país de origen, dando a entender implícitamente que no habría una diferencia sustancial entre el país como estado-nación (p. ej., España) o como pueblo (p. ej., españoles), de modo que sería necesario declarar con precisión cuál es el grupo de referencia utilizado en cada estudio, ya que en los diferentes contextos culturales el nivel de fusión de identidad con el estado-nación podría ser distinto al nivel de fusión con el pueblo; y, por último,

d. que al parecer la teoría de la fusión de identidad podría tener diferentes directrices que hay que seguir explorando, como sucede con la fusión interpersonal, la protofusión, la fusión en contextos migratorios - fusión con el país de origen y con el país de acogida - o la fusión con entidades que no necesariamente son grupos de personas - como un animal, marcas comerciales, figuras públicas, valores o ideales, entre otras-.

Teniendo esto en cuenta, se puede concluir que la teoría de la fusión de identidad ha avanzado significativamente en los últimos años, sin embargo, aún es necesaria la aplicación de nuevos estudios que permitan dar mayor sostén empírico a la teoría, de modo que, gracias a las limitaciones antes señaladas, son múltiples líneas de investigación a desarrollar o a seguir desarrollando.

\section{Referencias}

Ato, M., López, J. J., \& Benavente, A. (2013). Un sistema de clasificación de los diseños de investigación en psicología. Anales de psicología, 29(3), 1038-1059. http://dx.doi. org/10.6018/analesps.29.3.178511

*Besta, T. (2014). Overlap between personal and group identity and its relation with radical pro-group attitudes: data from a central european cultural context. Studia Psychologica, 56(1), 67-81. https://doi.org/10.21909/sp.2014.01.651

*Besta, T. (2018). Independent and interdependent? Agentic and communal? Self-construals of people fused with a group. Anales de Psicología, 34(1), 123-134. http://dx.doi. org/10.6018/analesps.34.1.266201

*Besta, T., \& Kossakowski, R. (2018). Football supporters: Group identity, perception of in-group and out-group members and pro-group action tendencies. Revista de Psicología del Deporte, 27(2), 15-22. https://www.rpd-online.com/ article/view/v27-n2-besta-kossakowski

*Besta, T., Gómez, Á., \& Vázquez, A. (2014). Readiness to deny group's wrongdoing and willingness to fight for its members: The role of poles' identity fusion with the country and religious group. Current Issues in Personality Psychology, 2(1), 49-55. https://doi.org/10.5114/cipp.2014.43101

*Besta, T., Jaśkiewicz, M., Kosakowska-Berezecka, N., Lawendowski, R., \& Zawadzka, A. M. (2018). What do I gain from joining crowds? Does self-expansion help to explain the relationship between identity fusion, group efficacy and collective action?. European Journal of Social Psychology, 48(2), O152-O167. https://doi.org/10.1002/ ejsp. 2332

*Besta, T., Kaźmierczak, M., \& Błażek, M. (2013). Identity fusion and status of the evaluator as moderators of 
self-enhancement and self-verification at the group level of self-description. Polish Psychological Bulletin, 44(4), 371378. https://doi.org/10.2478/ppb-2013-0040

*Besta, T., Mattingly, B., \& Błażek, M. (2015). When membership gives strength to act: Inclusion of the group into the self and feeling of personal agency. The Journal of social psychology, 156(1), 56-73. https://doi.org/10.1080/002245 45.2015.1053838

*Besta, T., Szulc, M., \& Jaśkiewicz, M. (2015). Political extremism, group membership and personality traits: who accepts violence?/Extremismo político, pertenencia al grupo y rasgos de personalidad: ¿Quién acepta la violencia?. Revista de Psicología Social, 30(3), 563-585. https://doi.or $\mathrm{g} / 10.1080 / 02134748.2015 .1065085$

Bélanger, J. J., Caouette, J., Sharvit, K., \& Dugas, M. (2014). The psychology of martyrdom: Making the ultimate sacrifice in the name of a cause. Journal of Personality and Social Psychology, 107(3), 494-515. http://dx.doi.org/10.1037/ a 0036855

Bélanger, J., Schumpe, B., Menon, B., Ng, J. \& Nociti, N. (2018). Self-sacrifice for a cause: a review and an integrative model. En V. Zeigler-Hill \& T. K. Shackelford, The sage handbook of personality and individual differences (pp. 465-485). SAGE Publications Ltd. http://dx.doi. org/10.4135/9781526451200.n25

Buhrmester, M. D., \& Swann, W.B., Jr. (2015). Identity fusion. En R. A. Scott \& S. M. Kosslyn (Eds.), Emerging Trends in the Social and Behavioral Sciences: An Interdisciplinary, Searchable, and Linkable Resource (pp. 1-15). Sage Publications. https://doi.org/10.1002/9781118900772. etrds0172

*Buhrmester, M. D., Burnham, D., Johnson, D., Curry, O. S., Macdonald, D., \& Whitehouse, H. (2018). How moments become movements: Shared outrage, group cohesion, and the lion that went viral. Frontiers in Ecology and Evolution, 6, 54. https://doi.org/10.3389/fevo.2018.00054

*Buhrmester, M. D., Fraser, W. T., Lanman, J. A., Whitehouse, H., \& Swann, Jr., W. B. (2015). When terror hits home: Identity fused americans who saw Boston bombing victims as "family" provided aid. Self and Identity, 14(3), 253-270. https://doi.org/10.1080/15298868.2014.992465

*Buhrmester, M. D., Gómez, Á., Brooks, M. L., Morales, J. F., Fernández, S., \& Swann Jr, W. B. (2012). My group's fate is my fate: Identity-fused Americans and Spaniards link personal life quality to outcome of 2008 elections. Basic and Applied Social Psychology, 34(6), 527-533. https://doi.org/ 10.1080/01973533.2012.732825

*Buhrmester, M. D., Newson, M., Vázquez, A., Hattori, W. T., \& Whitehouse, H. (2018). Winning at any cost: Identity fusion, group essence, and maximizing ingroup advantage. Self and Identity, 17(5), 500-516. https://doi.org/10.1080/1 5298868.2018.1452788

*Carnes, N. C., \& Lickel, B. (2018). Moral binding: How emotions, convictions, and identity fusion shape progroup behavior. Self and Identity, 17(5), 549-573. https://doi.org/1 0.1080/15298868.2018.1451362

*Dahling, J. J., \& Gutworth, M. B. (2017). Loyal rebels? A test of the normative conflict model of constructive deviance. Journal of Organizational Behavior, 38(8), 1167-1182. https://doi.org/10.1002/job.2194

*Fredman, L. A., Bastian, B., \& Swann, W. B, Jr. (2017). God or country? Fusion with Judaism predicts desire for retaliation following Palestinian stabbing Intifada. Social Psychological and Personality Science, 8(8), 882-887. https://doi.org/10.1177/1948550617693059

Fredman, L. A., Buhrmester, M. D., Gomez, A., Fraser, W. T., Talaifar, S., Brannon, S. M., \& Swann, W. B, Jr.. (2015). Identity fusion, extreme pro-group behavior, and the path to defusion. Social and Personality Psychology Compass, 9(9), 468-480. https://doi.org/10.1111/spc3.12193

*Gaviria, E., Ferreira, C. M., Martínez, M., \& Whitehouse, H. (2015). Identity and the developmental origins of fusion: An exploratory approach. Revista de Psicología Social: International Journal of Social Psychology, 30(3), 481-511. https://doi.org/10.1080/02134748.2015.1065088

Gómez, Á., \& Vázquez, A. (2015). El poder de 'sentirse uno' con un grupo: fusión de la identidad y conductas progrupales extremas. Revista de Psicología Social, 30(3), 1-31. https://doi.org/10.1080/02134748.2015.1065089

Gómez, Á., Brooks, M. L., Buhrmester, M. D., Vázquez, A., Jetten, J., \& Swann Jr, W. B. (2011). On the nature of identity fusion: Insights into the construct and a new measure. Journal of Personality and Social Psychology, 100(5), 918933. http://dx.doi.org/10.1037/a0022642

*Gómez, Á., Morales, J. F., Hart, S., Vázquez, A., \& Swann, Jr., W. B. (2011). Rejected and excluded forevermore, but even more devoted: Irrevocable ostracism intensifies loyalty to the group among identity fused persons. Personality and Social Psychology Bulletin, 37(12), 1574-1586. https://doi. org/10.1177/0146167211424580

Gómez, Á., Vázquez, A., López-Rodríguez, L., Talaifar, S., Martínez, M., Buhrmester, M. D., \& Swann Jr, W. B. (2019). Why people abandon groups: Degrading relational vs collective ties uniquely impacts identity fusion and identification. Journal of Experimental Social Psychology, 85, 103853. https://doi.org/10.1016/j.jesp.2019.103853

*Grinde, B., Nes, R. B., MacDonald, I. F., \& Wilson, D. S. (2018). Quality of life in intentional communities. Social Indicators Research, 137(2), 625-640. https://doi. org/10.1007/s11205-017-1615-3

*Heger, A. K., \& Gaertner, L. (2018). Testing the identity synergy principle: Identity fusion promotes self and group sacrifice. Self and Identity, 17(5), 487-499. https://doi.org/10. 1080/15298868.2017.1422538

Henrich, J., Heine, S. J., \& Norenzayan, A. (2010). Most people are not WEIRD. Nature, 466(7302), 29. https://www.nature.com/articles/466029a 
*Howard, M. C., \& Magee, S. M. (2013). To boldly go where no group has gone before: An analysis of online group identity and validation of a measure. Computers in Human Behavior, 29(5), 2058-2071. http://dx.doi.org/10.1016/j. chb.2013.04.009

*Jaśkiewicz, M., \& Besta, T. (2014). Is easy access related to better life? Walkability and overlapping of personal and communal identity as predictors of quality of life. Applied research in quality of life, 9(3), 505-516. https://doi. org/10.1007/s11482-013-9246-6

Jiménez, J., Gomez, A., Buhrmester, M. D., Vázquez, A., Whitehouse, H., \& Swann, W. B. (2016). The dynamic identity fusion index: A new continuous measure of identity fusion for web-based questionnaires. Social Science Computer Review, 34(2), 215-228. https://doi. org/10.1177/0894439314566178

*Jong, J., Whitehouse, H., Kavanagh, C., \& Lane, J. (2015). Shared negative experiences lead to identity fusion via personal reflection. PloS one, 10(12), e0145611. https://doi. org/10.1371/journal.pone.0145611

*Joo, M., \& Park, S. W. (2017). Effect of identity fusion on decision to make extreme sacrifices in romantic relationships: The moderating role of impulsiveness. British Journal of Social Psychology, 56(4), 819-827. https://doi.org/10.1111/ bjso. 12218

*Kossakowski, R., \& Besta, T. (2018). Football, conservative values, and a feeling of oneness with the group: a study of Polish football fandom. East European Politics and Societies, 32(4), 866-891. https://doi.org/10.1177/0888325418756991

*Kunst, J. R., Boos, B., Kimel, S. Y., Obaidi, M., Shani, M., \& Thomsen, L. (2018). Engaging in extreme activism in support of others' political struggles: The role of politically motivated fusion with out-groups. PloS one, 13(1), e0190639. https://doi.org/10.1371/journal.pone.0190639

Liberati, A., Altman, D. G., Tetzlaff, J., Mulrow, C., Gotzsche, P. C., Ioannidis, J. P., Clarke, M., Deveraux, P. J., Kleijnen, J., \& Moher, D. (2009). The PRISMA statement for reporting systematic reviews and meta-analyses of studies that evaluate health care interventions: explanation and elaboration. Journal of clinical epidemiology, 62(10), e1-e34. https://doi.org/10.1016/j.jclinepi.2009.06.006

*Lin, J. S., \& Sung, Y. (2014). Nothing can tear us apart: The effect of brand identity fusion in consumer-brand relationships. Psychology \& Marketing, 31(1), 54-69. https://doi. org/10.1002/mar.20675

*Misch, A., Fergusson, G., \& Dunham, Y. (2018). Temporal dynamics of partisan identity fusion and prosociality during the 2016 US presidential election. Self and Identity, 17(5), 531-548. https://doi.org/10.1080/15298868.2018.1430063

Montero, I., \& León, O. G. (2007). A guide for naming research studies in Psychology. International Journal of Clinical and Health Psychology, 7(3), 847-862. http://www.redalyc.org/ articulo.oa? $\mathrm{id}=33770318$
*Newson, M., Bortolini, T., Buhrmester, M., da Silva, S. R., da Aquino, J. N., \& Whitehouse, H. (2018). Brazil's football warriors: Social bonding and inter-group violence. Evolution and Human Behavior, 39(6), 675-683. https://doi. org/10.1016/j.evolhumbehav.2018.06.010

*Newson, M., Buhrmester, M., \& Whitehouse, H. (2016). Explaining lifelong loyalty: The role of identity fusion and self-shaping group events. PloS one, 11(8), e0160427. https://doi.org/10.1371/journal.pone.0160427

*Páez, D., Rimé, B., Basabe, N., Wlodarczyk, A., \& Zumeta, L. (2015). Psychosocial effects of perceived emotional synchrony in collective gatherings. Journal of Personality and Social Psychology, 108(5), 711-729. https://doi. org/10.1037/pspi0000014

*Paredes, B., Briñol, P., \& Gómez, Á. (2018). Identity fusion leads to willingness to fight and die for the group: The moderating impact of being informed of the reasons behind other members' sacrifice. Self and Identity, 17(5), 517-530. https://doi.org/10.1080/15298868.2017.1419503

*Reddish, P., Tong, E. M., Jong, J., Lanman, J. A., \& Whitehouse, H. (2016). Collective synchrony increases prosociality towards non-performers and outgroup members. British Journal of Social Psychology, 55(4), 722-738. https://doi.org/10.1111/bjso.12165

Robert, L., Virpi, L., \& John, L. (2019). Self sacrifice and kin psychology in war: threats to family predict decisions to volunteer for a women's paramilitary organization. Evolution and Human Behavior, 40(6), 543-550. https://doi. org/10.1016/j.evolhumbehav.2019.06.001

*Segal, K., Jong, J., \& Halberstadt, J. (2018). The fusing power of natural disasters: An experimental study. Self and Identity, 17(5), 574-586. https://doi.org/10.1080/15298868. 2018.1458645

*Semnani-Azad, Z., Sycara, K., \& Lewis, M. (2012). "Dynamics of helping behavior and cooperation across culture", 2012 International Conference on Collaboration Technologies and Systems (CTS), Denver, Colorado, pp. 525-530. http:// dx.doi.org/10.1109/CTS.2012.6261100

*Sheikh, H., Atran, S., Ginges, J., Wilson, L., Obeid, N., \& Davis, R. (2014). The devoted actor as parochial altruist: Sectarian morality, identity fusion, and support for costly sacrifices. Cliodynamics, 5(1), 23-40. https://doi. org/10.21237/C7clio5124901

*Sheikh, H., Gómez, Á., \& Atran, S. (2016). Empirical evidence for the devoted actor model. Current Anthropology, 57(S13), S204-S209. https://doi.org/10.1086/686221

*Steffens, N. K., Peters, K., Haslam, S. A., \& van Dick, R. (2017). Dying for charisma: Leaders' inspirational appeal increases post-mortem. The Leadership Quarterly, 28(4), 530-542. https://doi.org/10.1016/j.leaqua.2016.09.001

Swann W. B., Jr., \& Buhrmester, M. D. (2015). Identity fusion. Current Directions in Psychological Science, 24(1), 52-57. https://doi.org/10.1177/0963721414551363 
*Swann W. B., Jr., Buhrmester, M. D., Gómez, A., Jetten, J., Bastian, B., Vázquez, A., Ariyanto, A., Besta, T., Christ, O., Cui, L., Finchilescu, G., González, R., Goto, N., Hornsey, M., Sharma, S., Susianto, H., \& Zhang, A. (2014). What makes a group worth dying for? Identity fusion fosters perception of familial ties, promoting self-sacrifice. Journal of personality and social psychology, 106(6), 912-926. https:// doi.org/10.1037/a0036089

*Swann, W. B., Jr., Gómez, Á., Buhrmester, M. D., LópezRodríguez, L., Jiménez, J., \& Vázquez, A. (2014). Contemplating the ultimate sacrifice: Identity fusion channels pro-group affect, cognition, and moral decision making. Journal of personality and social psychology, 106(5), 713727. https://doi.org/10.1037/a0035809

*Swann, W. B., Jr., Gómez, Á., Dovidio, J. F., Hart, S., \& Jetten, J. (2010). Dying and killing for one's group: Identity fusion moderates responses to intergroup versions of the trolley problem. Psychological Science, 21(8), 1176-1183. https:// doi.org/10.1177/0956797610376656

*Swann, W. B., Jr., Gómez, Á., Huici, C., Morales, F., \& Hixon, J. G. (2010). Identity fusion and self-sacrifice: Arousal as a catalyst of pro-group fighting, dying, and helping behavior. Journal of Personality and Social Psychology, 99(5), 824841. https://doi.org/10.1037/a0020014

*Swann, W. B., Jr., Gómez, Á., Seyle, D. C., Morales, J., \& Huici, C. (2009). Identity fusion: The interplay of personal and social identities in extreme group behavior. Journal of Personality and Social Psychology, 96(5), 995-1011. https://doi.org/10.1037/a0013668

*Swann, W. B., Jr., Gómez, Á., Vázquez, A., Guillamón, A., Segovia, S., \& Carillo, B. (2015). Fusion with the crossgender group predicts genital sex reassignment surgery. Archives of sexual behavior, 44(5), 1313-1318. https://doi. org/10.1007/s10508-014-0470-4

Swann, W. B., Jr., Jetten, J., Gómez, Á., Whitehouse, H., \& Bastian, B. (2012). When group membership gets personal: A theory of identity fusion. Psychological review, 119(3), 441-456. https://doi.org/10.1037/a0028589

Tajfel, H., \& Turner, J. C. (1979). An integrative theory of intergroup conflict. En W. G. Austin \& S. Worchel (Eds.), The social psychology of intergroup relations (pp. 33-47). Brooks-Cole.

Urrútia, G., \& Bonfill, X. (2010). Declaración PRISMA: una propuesta para mejorar la publicación de revisiones sistemáticas y metaanálisis. Medicina clínica, 135(11), 507-511. https://doi.org/10.1016/j.medcli.2010.01.015
*Van Dick, R., Fink, L., Steffens, N. K., Peters, K., \& Haslam, S. A. (2018). Attributions of leaders' charisma increase after their death: The mediating role of identity leadership and identity fusion. Leadership. https://doi. org/10.1177/1742715018807042

*Vázquez, A., Gómez, Á., \& Swann, W. B.. Jr. (2017). Do historic threats to the group diminish identity fusion and its correlates?. Self and Identity, 16(4), 480-503. https://doi.or $\mathrm{g} / 10.1080 / 15298868.2016 .1272485$

*Vázquez, A., Gómez, Á., Ordoñana, J. R., \& Paredes, B. (2015). Desde la fusión interpersonal a la fusión extendida: Relaciones entre la fusión con los hermanos y la fusión con el país. Revista de Psicología Social, 30(3), 512-530. http:// dx.doi.org/10.1080/02134748.2015.1093755

*Vázquez, A., Gómez, Á., Ordoñana, J. R., Swann, W. B., \& Whitehouse, H. (2017). Sharing genes fosters identity fusion and altruism. Self and Identity, 16(6), 684-702. https:// doi.org/10.1080/15298868.2017.1296887

*Walsh, C. M., \& Neff, L. A. (2018). We're better when we blend: The benefits of couple identity fusion. Self and Identity, 17(5), 587-603. https://doi.org/10.1080/15298868 .2018.1430062

Whitehouse, H., \& Lanman, J. A. (2014). The Ties That Bind Us: Ritual, Fusion, and Identification. Current Anthropology, 55(6), 674-695. https://doi.org/10.1086/678698

*Whitehouse, H., McQuinn, B., Buhrmester, M., \& Swann, W. B. (2014). Brothers in Arms: Libyan revolutionaries bond like family. Proceedings of the National Academy of Sciences, 111(50), 17783-17785. https://doi.org/10.1073/ pnas. 1416284111

*Yoo, J., Swann, W. B., \& Kim, K. (2014). The influence of identity fusion on patriotic consumption: A cross-cultural comparison of Korea and the US. The Korean Journal of Advertising, 25(5), 81-104. https://doi.org/10.14377/ kja.2014.7.15.81

Yuan, C., Tao, S., \& Lu, H. (2014). Review of Identity Fusion. Advances in Psychology, 4(7), 914-922. http://dx.doi. org/10.12677/AP.2014.47118

*Zumeta, L. N., Oriol, X., Telletxea, S., Amutio, A., \& Basabe, N. (2016). Collective efficacy in sports and physical activities: Perceived emotional synchrony and shared flow. Frontiers in psychology, 6, 1960. https://doi.org/10.3389/ fpsyg.2015.01960

*Zumeta, L., Basabe, N., Wlodarczyk, A., Bobowik, M., \& Páez, D. (2016). Shared flow and positive collective gatherings. Anales de Psicología, 32(3), 717-727. http://dx.doi. org/10.6018/analesps.32.3.261651 